\title{
The contact problem in Lagrangian systems subject to bilateral and unilateral constraints, with or without sliding Coulomb's friction: a tutorial
}

\author{
Alejandro Blumentals ${ }^{1} \cdot$ Bernard Brogliato $^{1}$. \\ Florence Bertails-Descoubes ${ }^{1}$
}

\begin{abstract}
This work deals with the existence and uniqueness of the acceleration and contact forces for Lagrangian systems subject to bilateral and/or unilateral constraints with or without sliding Coulomb's friction. Sliding friction is known to yield singularities in the system, such as Painlevé's paradox. Our work aims at providing sufficient conditions on the parameters of the system so that singularities are avoided (i.e., the contact problem is at least solvable). To this end, the frictional problem is treated as a perturbation of the frictionless case. We provide explicit criteria, in the form of calculable upper bounds on the friction coefficients, under which the frictional contact problem is guaranteed to remain well-posed. Complementarity problems, variational inequalities, quadratic programs and inclusions in normal cones are central tools.
\end{abstract}

Keywords Lagrangian systems · Unilateral constraint · Bilateral constraint · Complementarity problem · Coulomb's friction · Gauss' principle $\cdot$ KKT conditions

\section{Introduction}

Lagrangian systems subject to (frictional) bilateral and unilateral constraints are considered. Such systems, mathematically described in Eqs. (1a)-(1d), feature a very rich dynamics because they are nonlinear, nonsmooth, and set-valued. A large number of studies have been led on their well-posedness. Their goal is to formulate conditions under which (1a)(1d) possesses a solution, that is, a trajectory $(q(\cdot), \dot{q}(\cdot))$ belonging to a certain functional space (with absolutely continuous positions and right-continuous velocities of local bounded variations) and satisfying (1a)-(1d) for all $t \geq 0$. It also consists in determining whether a solution is unique for given initial data $(q(0), \dot{q}(0))$, whether solutions depend continuously on initial data or not, whether they converge to some equilibrium state (stability and control analysis [36]), et cetera.

\footnotetext{
B. Brogliato

bernard.brogliato@inria.fr

1 INRIA Grenoble Rhône-Alpes and Laboratoire Jean Kuntzman, University Grenoble-Alpes, 655 avenue de l'Europe, 38334 Saint-Ismier, France
} 
Besides such analysis, one may in turn be interested in properties of least mathematical relevance but of high interest for mechanical engineers. Typically, are the contact forces calculable during persistent motion phases? Are they calculable in a unique way? If some solvability results can be obtained in the absence of friction, what happens when Coulomb friction is added at some contacts? More generally, how does the system evolve during persistent contact phases in the presence of friction, for example, does the dynamics exhibit some singular states? In multibody systems dynamics, these questions are to be examined by studying the so-called contact problem, which assumes the mechanical state $(q(t), \dot{q}(t))$ to be known at a given time $t$ and considers the acceleration $\ddot{q}(t)$ and the contact forces at time $t$ as unknowns. It thus results in a merely algebraic system, for which well-posedness remains a difficult question. Studying the contact problem is of high interest in multibody systems, where one often wants to calculate the contact forces at a certain given time. Another motivation comes from event-driven numerical integration methods, where one has to solve the contact problem at a given time where possibly the state of the system may switch to another mode [1].

In this paper, our goal is to study conditions under which the contact problem is well posed. We first focus ourselves on frictionless systems, then on systems with sliding friction (single-valued law). Indeed, sliding friction is known to yield hard singularities like the Painlevé paradoxes [4, 21, 47], whereas it has been shown in the seminal paper [48] that sticking modes can always, under some mild assumption, be continued in another contact mode (including detachment from the constraints).

For systems subject to bilateral constraints, necessary and sufficient conditions for the existence and uniqueness of the acceleration in the presence of a singular mass matrix and redundant constraints are given in $[31,59]$. The computation of constraint reactions in the redundant case is addressed via augmented Lagrangian methods in $[5,7,18,52]$ by a constraint elimination method combined with solvability analysis techniques in [20, 61, 63] and by a pseudo-inverse method in [58]. Although the mechanical and geometrical interpretation of the augmented Lagrangian method is well understood [7], only recently its convergence and robustness properties have been proved in the absence of a linearly independent constraint qualification hypothesis [28]. In the case of bilateral constraints with sliding Coulomb's friction, Matrosov and Finogenko derive an implicit criterion in [41, 42], which guarantees the uniqueness of the acceleration for small enough friction coefficients; however, no explicit upper bounds are given. In [4], such an upper bound may be found but concerns only systems with a single contact point. For systems subject only to unilateral constraints and sliding Coulomb's friction, an existence result based on small enough friction coefficients and complementarity theory is given in [37], and an explicit upper bound is established in [48]. The contact problem with mixed (bilateral and unilateral) constraints has received surprisingly less attention. Its analysis in the frictionless case with redundant constraints and a singular mass matrix is given in [11], whereas in the frictional sliding case, it is established in [27] that it becomes a mixed linear complementarity problem but no explicit condition for its solvability is given.

\subsection{Dynamics of Lagrangian systems subject to bilateral, unilateral, and frictional constraints}

In a Lagrangian formalism, such systems may be written generically as follows: 


$$
\left\{\begin{array}{l}
M(q) \ddot{q}+F(q, \dot{q}, t) \\
\quad=\nabla h_{\mathrm{n}, b}(q) \lambda_{\mathrm{n}, b}+\nabla h_{\mathrm{n}, u}(q) \lambda_{\mathrm{n}, u}+H_{\mathrm{t}, b}(q) \lambda_{\mathrm{t}, b}+H_{\mathrm{t}, u}(q) \lambda_{\mathrm{t}, u}, \\
\text { Sliding friction (Coulomb): } \quad \lambda_{\mathrm{t}, i}=-\mu_{i}\left|\lambda_{\mathrm{n}, i}\right| \frac{v_{\mathrm{t}, i}}{\left\|v_{\mathrm{t}, i}\right\|}, \quad v_{\mathrm{t}, i} \neq 0, \quad 1 \leq i \leq m, \\
\text { Complementarity conditions: } \quad 0 \leq h_{\mathrm{n}, u}(q) \perp \lambda_{\mathrm{n}, u} \geq 0, \\
\text { Bilateral (holonomic) constraints: } \quad h_{\mathrm{n}, b}(q)=0,
\end{array}\right.
$$

where $q \in \mathbb{R}^{n}$ is the vector collecting the generalized coordinates $q_{i}, 1 \leq i \leq n$, assumed to be independent when all the constraints are removed, $\dot{q}$ is the vector of generalized velocities, $M(q)=M(q)^{T}$ is the inertia matrix, always assumed to be at least positive semidefinite (it may be assumed nonsingular in some cases), and $F(q, \dot{q}, t)$ collects internal forces (including forces deriving from a potential, plus Coriolis and centrifugal forces) and external actions on the system such as disturbances or control.

We consider $m=m_{u}+m_{b}$ constraints (or contacts) consisting of $m_{u}$ unilateral (inequality) constraints $h_{\mathrm{n}, u}(q) \in \mathbb{R}^{m_{u}}$ and $m_{b}$ bilateral (equality) constraints $h_{\mathrm{n}, b}(q) \in \mathbb{R}^{m_{b}}$. The matrix $\nabla h_{n, u}(q)$ (respectively $\left.\nabla h_{n, b}(q)\right)$ collects on each column the gradient for each unilateral constraint (for each bilateral constraint, respectively). The vectors of Lagrange multipliers $\lambda_{\mathrm{n}, u} \in \mathbb{R}^{m_{u}}$ and $\lambda_{\mathrm{n}, b} \in \mathbb{R}^{m_{b}}$ are associated with the unilateral and bilateral constraints, respectively. From a mechanical point of view, $\lambda_{\mathrm{n}, u}$ and $\lambda_{\mathrm{n}, b}$ correspond to the normal components of the contact forces in the unilateral and bilateral cases, respectively, and are obtained from the local contact kinematics [23, Chap. 10], [1, Chap. 3]. The unilateral constraints and their associated Lagrange multipliers are related through the complementarity condition (1c), which is to be understood componentwise (per contact). It models the fact that for each contact $i$, the normal contact force should not act at a unilateral contact point if the contact is open (i.e., $h_{\mathrm{n}, u, i}(q)>0$ implies $\lambda_{\mathrm{n}, u, i}=0$ ), and that $\lambda_{\mathrm{n}, u, i}>0$ if and only if $h_{\mathrm{n}, u, i}(q)=0$. The matrices $H_{\mathrm{t}, b}(q)$ and $H_{\mathrm{t}, u}(q)$ map the local tangential velocities to generalized velocities (see Sect. 3.1 for more details).

The coefficients of friction at each contact point $i$ are $\mu_{i} \geq 0$. We consider in this study frictional contacts in a sliding mode only (nonzero relative tangential velocities). Also, the contact problem (with unknowns the acceleration and contact forces) is considered at a given time instant. This means that by a proper choice of the local contact kinematics frames we have $\frac{v_{\mathrm{t}, i}}{\left\|v_{\mathrm{t}, i}\right\|}=\operatorname{sgn}\left(v_{\mathrm{t}, i}\right)$, both for two-dimensional and three-dimensional friction.

The article is organized as follows. We first review in detail the frictionless case in Sect. 2, where results for the bilaterally and unilaterally constrained cases are recalled. In Sect. 3, we show how the problem gets all the more complex as Coulomb's friction is considered and as mixed constraints (both bilateral and unilateral) are added. Conclusions end the paper, and some useful results are recalled in Appendices A-E.

\subsection{Further notations}

Mathematical notations $\quad \mathbb{R}^{n}$ is the set of $n$-vectors with real entries, and $\mathbb{R}_{+}^{n}$ is the set of $n$ vectors with nonnegative entries. Let $a_{1}, a_{2}, \ldots, a_{n}$ be some given reals, then $[a]=\operatorname{diag}\left(a_{i}\right)$ is the $n \times n$ diagonal matrix with entries $a_{i}$. For a real square matrix $A \in \mathbb{R}^{n \times n}$, its induced norm is $\|A\|_{2}=\max _{x \in \mathbb{R}^{n},\|x\|_{2}=1}\|A x\|_{2}$, where $\|x\|_{2}$ is the Euclidean norm on the vector space $\mathbb{R}^{n}$. This induced matrix norm is sometimes denoted as $\|A\|_{2,2}$. We have $\|A\|_{2}=\sigma_{\max }(A)=\sqrt{\lambda_{\max }\left(A A^{T}\right)}$ [6, Proposition 9.4.7], where $\sigma_{\max }$ is the maximum singular value of $A$, and $\lambda_{\min }(\cdot)$ and $\lambda_{\max }(\cdot)$ are its smallest and largest eigenvalues, respectively [6, Proposition 9.4.7]. Moreover, if $A$ is invertible, then $\sigma_{\min }(A)=\frac{1}{\sigma_{\max }\left(A^{-1}\right)}$, where $\sigma_{\min }(A)$ 
is the smallest singular value of $A$ [6, Fact 6.3.21]. A positive definite (resp. semidefinite) matrix is denoted $A \succ 0$ (resp. $A \succeq 0$ ); it may be nonsymmetric. Let $f: \mathbb{R}^{n} \mapsto \mathbb{R}^{p}$ be a differentiable function. Its Euclidean gradient is $\nabla f(x)=\left(\nabla f_{1}(x) \nabla f_{2}(x) \ldots \nabla f_{p}(x)\right) \in$ $\mathbb{R}^{n \times p}$, and its Jacobian is $\frac{\partial f}{\partial x}(x)=\nabla f(x)^{T}$. The cardinality of a countable set $\mathcal{I}$ is denoted $\operatorname{card}(\mathcal{I})$. For a set $S \subseteq \mathbb{R}^{n}$, its orthogonal complement is the subspace $S^{\perp} \triangleq\{x \in$ $\mathbb{R}^{n} \mid x^{T} y=0$ for all $\left.y \in S\right\}$, and its boundary is denoted $\operatorname{bd}(S)$. We have $\operatorname{Ker}(A)=\operatorname{Im}\left(A^{T}\right)^{\perp}$ and $\operatorname{Ker}(A)=\operatorname{Im}\left(A^{T}\right)^{\perp}$ for any matrix $A$.

Mechanical notations Since contacts may be frictionless, or in contrast may involve Coulomb friction, we adopt the following conventions:

- Bilateral contacts: $1 \leq i \leq m_{b}$, i.e., $i \in \mathcal{I}_{b}$.

- Frictional bilateral contacts (sliding): $1 \leq i \leq m_{b}^{\mu}$, i.e., $i \in \mathcal{I}_{b}^{\mu}$.

- Frictionless bilateral contacts: $m_{b}^{\mu}+1 \leq i \leq m_{b}$, i.e., $i \in \mathcal{I}_{b}^{0}$.

- Unilateral contacts: $m_{b}+1 \leq i \leq m$, i.e., $i \in \mathcal{I}_{u}$.

- Frictional unilateral contacts (sliding): $m_{b}+1 \leq i \leq m_{u}^{\mu}$, i.e., $i \in \mathcal{I}_{u}^{\mu}$.

- Frictionless unilateral contacts: $m_{u}^{\mu}+1 \leq i \leq m$, i.e., $i \in \mathcal{I}_{u}^{0}$.

We may therefore rewrite the first line in (1a)-(1d) as

$$
\begin{aligned}
M(q) \ddot{q}+F(q, \dot{q}, t)= & \sum_{i \in \mathcal{I}_{b}^{0}} \nabla h_{\mathrm{n}, b, i}^{0}(q) \lambda_{\mathrm{n}, b, i}^{0}+\sum_{i \in \mathcal{I}_{u}^{0}} \nabla h_{\mathrm{n}, u, i}^{0}(q) \lambda_{\mathrm{n}, u, i}^{0} \\
& +\sum_{i \in \mathcal{I}_{b}^{\mu}} \nabla h_{\mathrm{n}, b, i}(q) \lambda_{\mathrm{n}, b, i}+\sum_{i \in \mathcal{I}_{b}^{\mu}} H_{\mathrm{t}, b, i}(q) \lambda_{\mathrm{t}, b, i} \\
& +\sum_{i \in \mathcal{I}_{u}^{\mu}} \nabla h_{\mathrm{n}, u, i}(q) \lambda_{\mathrm{n}, u, i}+\sum_{i \in \mathcal{I}_{u}^{\mu}} H_{\mathrm{t}, u, i}(q) \lambda_{\mathrm{t}, u, i} .
\end{aligned}
$$

In the sequel, we usually drop the time argument to lighten the formulas.

\section{Frictionless systems}

This section starts with the classical KKT system for bilaterally constrained systems of index 1 and then elaborates on how this system is transformed when unilateral constraints are added. A thorough analysis of the ubiquitous KKT system is proposed in Appendix E.

\subsection{Bilaterally constrained systems}

Let us assume that $m_{u}=0$, that is, $\mathcal{I}_{u}^{0}=\emptyset$, and that $\mathcal{I}_{b}^{0} \neq \emptyset$. The Lagrangian system (1a)(1d) boils down to a differential-algebraic equation (DAE) of index $3[24,56]$,

$$
\left\{\begin{array}{l}
M(q) \ddot{q}+F(q, \dot{q}, t)=\nabla h_{\mathrm{n}, b}(q) \lambda_{\mathrm{n}, b}, \\
h_{\mathrm{n}, b}(q)=0 .
\end{array}\right.
$$

Reducing the index to 1 consists in differentiating twice the constraint $h_{\mathrm{n}, b}(q)=0$ to obtain $\ddot{h}_{\mathrm{n}, b}=\nabla h_{\mathrm{n}, b}(q)^{T} \ddot{q}+\frac{d}{d t}\left(\nabla h_{\mathrm{n}, b}(q)^{T}\right) \dot{q}=0$. This new equality, combined with the dynamics, 
yields the following linear system:

$$
\underbrace{\left(\begin{array}{cc}
M(q) & -\nabla h_{\mathrm{n}, b}(q) \\
\nabla h_{\mathrm{n}, b}(q)^{T} & 0
\end{array}\right)}_{\triangleq_{M_{b}(q)}}\left(\begin{array}{c}
\ddot{q} \\
\lambda_{\mathrm{n}, b}
\end{array}\right)=\left(\begin{array}{c}
-F(q, \dot{q}, t) \\
-\frac{d}{d t}\left(\nabla h_{\mathrm{n}, b}(q)^{T}\right) \dot{q}
\end{array}\right) .
$$

Time-varying constraints $h_{\mathrm{n}, b}(q, t)=0$ may be considered in the analysis. Then

$$
\frac{d}{d t} h_{\mathrm{n}, b}(q, t)=\nabla h_{\mathrm{n}, b}(q, t)^{T} \dot{q}(t)+\frac{\partial h_{\mathrm{n}, b}}{\partial t}(q, t)
$$

and

$$
\begin{aligned}
\frac{d^{2}}{d t^{2}} h_{\mathrm{n}, b}(q, t)= & \nabla h_{\mathrm{n}, b}(q, t)^{T} \ddot{q}(t)+\frac{d}{d t}\left(\nabla h_{\mathrm{n}, b}(q, t)^{T}\right) \dot{q}(t)+\frac{\partial}{\partial q} \frac{\partial h_{\mathrm{n}, b}}{\partial t}(q, t) \dot{q}(t) \\
& +\frac{\partial^{2} h_{\mathrm{n}, b}}{\partial t^{2}}(q, t) .
\end{aligned}
$$

If needed, it is thus sufficient to add the missing terms to step from the time-invariant to the time-varying case. Recall that $M(q)$ is assumed to be symmetric positive semidefinite (with possibly $\operatorname{rank}(M(q))<n)$. The matrix in the left-hand side, denoted as $M_{b}(q)$ and often called the DAE matrix, is ubiquitous not only in the analysis and numerics of Lagrangian systems with holonomic constraints [24, 31, 34, 56, 57, 59, 62], but also in convex quadratic minimization. In the latter context, the $M_{b}(q)$ matrix is referred to as the KKT matrix, and system (4) corresponds to the KKT system of the following quadratic minimization problem $[8, \S 10.1 .1]$ :

$$
\begin{aligned}
& \ddot{q}(t)=\operatorname{argmin}_{z}\left(\frac{1}{2} z^{T} M(q) z+F(q, \dot{q}, t)^{T} z\right) \\
& \text { subject to } \nabla h_{\mathrm{n}, b}(q)^{T} z+\frac{d}{d t}\left(\nabla h_{\mathrm{n}, b}(q)^{T}\right) \dot{q}=0,
\end{aligned}
$$

which can be, in the case where $M(q)$ is nonsingular, interpreted mechanically as the Gauss principle of least constraints applied to a Lagrangian system subject to bilateral holonomic constraints [58].

The DAE or KKT matrix $M_{b}(q)$ is a positive semidefinite matrix. The fact that $M_{b}(q)$ has a skew-symmetric part in (4) is not intrinsic to the problem. Indeed, it stems from an arbitrary choice in the way the bilateral constraints are introduced in the dynamics (1a)-(1d) and thus from the choice of the sign of the multiplier. By changing the sign of the multiplier, the problem may be analyzed equivalently without the minus sign in $-\nabla h_{\mathrm{n}, b}(q)$, thus setting $M_{b}(q)$ symmetric (meanwhile causing the loss of positiveness for $M_{b}(q)$ ). This is actually the convention adopted in most of the DAE literature. The next proposition gathers solvability results for system (4) from Optimization [8, §10.1.1] and Mechanics [31].

Proposition 1 Consider the KKT system in (4).

(i) Let $m_{b}<n$ and $\nabla h_{\mathrm{n}, b}(q)$ have full (column) rank $m_{b}$. Then $M_{b}(q)$ is nonsingular (or equivalently, given $(q, \dot{q})$ and any arbitrary right-hand side vector of (4), there exists a unique solution $\left(\ddot{q}, \lambda_{\mathrm{n}, b}\right)$ to $\left.(4)\right)$ if and only if

$$
\operatorname{Ker}(M(q)) \cap \operatorname{Ker}\left(\nabla h_{\mathrm{n}, b}(q)^{T}\right)=\{0\} .
$$


Moreover, if $M(q) \succ 0$, then the multiplier $\lambda_{n b}$ can be computed in closed form by solving the linear equation

$$
A_{n b}(q) \lambda_{\mathrm{n}, b}+w_{b}(q, \dot{q}, t)=0,
$$

where $A_{n b}(q)=A_{n b}(q)^{T} \triangleq \nabla h_{\mathrm{n}, b}(q)^{T} M(q)^{-1} \nabla h_{\mathrm{n}, b}(q) \succ 0$, corresponding to the Schur complement ${ }^{1}$ of $M(q)$ in the DAE matrix $M_{b}(q)$, and where $w_{b}(q, \dot{q}, t)=$ $-\nabla h_{\mathrm{n}, b}(q)^{T} M(q)^{-1} F(q, \dot{q}, t)+\frac{d}{d t}\left(\nabla h_{\mathrm{n}, b}(q)^{T}\right) \dot{q}$. Injecting the expression $\lambda_{\mathrm{n}, b}=$ $-A_{n b}(q)^{-1} w_{b}(q, \dot{q}, t)$ in the dynamics then yields a dynamical system that sets the submanifold $\left\{(q, \dot{q}) \in \mathbb{R}^{n} \times \mathbb{R}^{n} \mid h_{\mathrm{n}, b}(q)=0\right.$ and $\left.\nabla h_{\mathrm{n}, b}(q)^{T} \dot{q}=0\right\}$ invariant.

(ii) Let $\nabla h_{\mathrm{n}, b}(q)$ have arbitrary rank and satisfy the compatibility of constraints, that is, $-\frac{d}{d t}\left(\nabla h_{\mathrm{n}, b}(q)^{T}\right) \dot{q} \in \operatorname{Im}\left(\nabla h_{\mathrm{n}, b}(q)^{T}\right)$. Then, given $(q, \dot{q})$ and an arbitrary force vector $F(q, \dot{q}, t)$,

- A solution $\left(\ddot{q}, \lambda_{\mathrm{n}, b}\right)$ of (4) exists, and

- The acceleration $\ddot{q}$ and the generalized contact force $\nabla h_{\mathrm{n}, b}(q) \lambda_{\mathrm{n}, b}$ are unique

if and only if condition (6) holds.

Proof Follows from the material in Appendix E (items (iii) (iii'), and (iv)).

Item (ii) obviously relies on weaker assumptions than item (i) since $\nabla h_{n b}(q)$ is not required to have full column rank $m_{b}$ but rather satisfies the compatibility of constraints as in [31]. In particular, we consider in (ii) cases where $m_{b}>n$, that is, where $M_{b}(q)$ is necessarily a singular matrix, and where some constraints are redundant. Note that if $M(q) \succ 0$, then the Schur complement satisfies $A_{n b}(q)=A_{n b}(q)^{T} \succeq 0$. Furthermore, we have $\operatorname{Ker}\left(A_{n b}(q)\right)=\operatorname{Ker}\left(\nabla h_{n b}(q)\right)$. In particular, $A_{n b}(q)$ is nonsingular if and only if $\nabla h_{n b}(q)$ has full column rank $m_{b} \leq n$. Singular mass matrices and redundant constraints are common features of multibody dynamical systems subject to bilateral constraints, due to rotation parameterization [55], or redundant generalized coordinates [59, 60].

\subsection{Unilaterally constrained systems}

Now we assume that all contacts are unilateral, $m_{b}=0$, that is, $\mathcal{I}_{b}=\emptyset$. Let us recall the so-called contact linear complementarity problem (contact LCP).

\subsubsection{Construction of the contact LCP}

Proposition 2 Let $h(\cdot)$ and $\lambda(\cdot)$ be two functions of time, and let $0 \leq h(t) \perp \lambda(t) \geq 0$ for all $t$. Assume that $h(\cdot)$ is absolutely continuous, that $\dot{h}(\cdot)$ is absolutely continuous in a right neighborhood of $t$, and that $\ddot{h}(\cdot)$ and $\lambda(\cdot)$ are right-continuous at time $t$.

(i) If $h(t)=0$, then $0 \leq \dot{h}(t) \perp \lambda(t) \geq 0$.

(ii) If $h(t)=0$ and $\dot{h}(t)=0$, then $0 \leq \ddot{h}(t) \perp \lambda(t) \geq 0$.

Proof (i) For any $t^{\prime} \geq t$, we have $h\left(t^{\prime}\right)-h(t)=\int_{t}^{t^{\prime}} \dot{h}(s) d s$. Suppose that $\dot{h}(t)<0$. Since $\dot{h}(\cdot)$ is right-continuous, there exists $\epsilon>0$ such that $\dot{h}(s)<0$ for all $s \in[t, t+\epsilon)$. Thus,

\footnotetext{
${ }^{1}$ The Schur complement of the invertible matrix $A_{11}$ in the $m \times n$ matrix $A=\left(\begin{array}{l}A_{11} A_{12} \\ A_{21} A_{22}\end{array}\right)$ is the matrix $A_{22}-$ $A_{21} A_{11}^{-1} A_{12}$. The Schur complement of the invertible matrix $A_{22}$ in $A$ is the matrix $A_{11}-A_{12} A_{22}^{-1} A_{21}$.
} 
for any $t^{\prime} \in[t, t+\epsilon)$, we have $h\left(t^{\prime}\right)<0$, which is impossible. Thus, we have $\dot{h}(t) \geq 0$. Now let $\dot{h}(t)>0$. By continuity there exists $\epsilon>0$ such that for all $t^{\prime} \in(t, t+\epsilon)$, we have $\dot{h}\left(t^{\prime}\right)>0$. Consequently, $h\left(t^{\prime}\right)>0$ for all $t^{\prime} \in(t, t+\epsilon)$, and $\lambda(t)=0$. Now suppose that $\lambda(t)>0$ and thus $h(t)=0$. Assume that $\dot{h}(t)>0$, so that $h\left(t^{\prime}\right)>0$ for all $t^{\prime} \in(t, t+\epsilon)$ and thus $\lambda\left(t^{\prime}\right)=0$ for all $t^{\prime} \in(t, t+\epsilon)$ : this is a contradiction, and consequently $\dot{h}(t) \leq 0$. From the nonnegativeness we infer $\dot{h}(t)=0$. Hence, (i) is proved. Part (ii) is proved in a similar way.

We see that Proposition 2 involves the lexicographical inequality $(h(t) \dot{h}(t) \ddot{h}(t)) \succcurlyeq 0 .^{2}$ Lexicographical inequalities are ubiquitous in the analysis of unilaterally constrained dynamical systems; see, for example, [2, 17, 23, 54]. Assume that $q(\cdot)$ and $h_{\mathrm{n}, u}(\cdot)$ are continuous, whereas $\dot{q}(\cdot), \ddot{q}(\cdot)$, and $\lambda_{\mathrm{n}, u}(\cdot)$ are right-continuous. Replacing $h(t)$ with $h_{\mathrm{n}, u, i} \circ q(t)$ and $\lambda(t)$ with $\lambda_{\mathrm{n}, u, i}(t)$ allows us to assert that $h_{\mathrm{n}, u, i}(q(t))=0$ implies that $0 \leq \nabla h_{\mathrm{n}, u, i}(q(t))^{T} \dot{q}(t) \perp \lambda_{\mathrm{n}, u, i}(t) \geq 0$, whereas $h_{\mathrm{n}, u, i}(q(t))=0$ and $\nabla h_{\mathrm{n}, u, i}(q(t))^{T} \dot{q}(t)=0$ imply $0 \leq \nabla h_{\mathrm{n}, u, i}(q(t))^{T} \ddot{q}(t)+w_{u, i}(q, \dot{q}, t) \perp \lambda_{\mathrm{n}, u, i}(t) \geq 0$, where $w_{u}(q, \dot{q}, t)$ is defined in (10). In fact, since we disregard impacts, we may even assume that $\dot{q}(\cdot)$ is continuous. In view of this, we have the following mixed $L C P(M L C P)$ whose unknown is the acceleration, and which is the counterpart of (4) for unilateral constraints:

$$
\left\{\begin{array}{l}
M(q) \ddot{q}+F(q, \dot{q}, t)=\nabla h_{\mathrm{n}, u}(q) \lambda_{\mathrm{n}, u}, \\
0 \leq \lambda_{\mathrm{n}, u} \perp \nabla h_{\mathrm{n}, u}(q)^{T} \ddot{q}+\frac{d}{d t}\left(\nabla h_{\mathrm{n}, u}(q)^{T}\right) \dot{q} \geq 0 .
\end{array}\right.
$$

\subsubsection{Analysis of the contact LCP}

In case $M(q) \succ 0$, the MLCP (8) is easily transformed by elimination of $\ddot{q}$ to construct the contact LCP that is the counterpart of (7):

Definition 1 (Frictionless Contact LCP) Let $\mu_{i}=0$ for all $1 \leq i \leq m, m_{b}=0, m_{u}>0$, and $M(q) \succ 0$. The frictionless contact LCP is given by

$$
0 \leq \lambda_{\mathrm{n}, u} \perp A_{n u}(q) \lambda_{\mathrm{n}, u}+w_{u}(q, \dot{q}, t) \geq 0,
$$

where $A_{n u}(q) \triangleq \nabla h_{\mathrm{n}, u}(q)^{T} M(q)^{-1} \nabla h_{\mathrm{n}, u}(q)$ is the Delassus matrix, and

$$
w_{u}(q, \dot{q}, t)=-\nabla h_{\mathrm{n}, u}(q)^{T} M(q)^{-1} F(q, \dot{q}, t)+\frac{d}{d t}\left(\nabla h_{\mathrm{n}, u}(q)^{T}\right) \dot{q} .
$$

If at a given time $t$ the solution of (9) satisfies $\lambda_{\mathrm{n}, u}(t)>0$, then the contact mode exists at $t$. If $\lambda_{\mathrm{n}, u}(t)=0$, then it may be that another mode has to be considered, depending on $w_{u}(q, \dot{q}, t)$ being $>0$ or null (componentwise). In a more general situation, it may occur that some components of the multiplier are positive whereas others are zero.

The next proposition gathers results from Moreau [43, 44], Lötstedt [38], Pang and Trinkle [49], and Brogliato and Goeleven [9, 11].

Proposition 3 (Frictionless, unilateral constraints) Let $\mu_{i}=0$ for all $1 \leq i \leq m, m_{b}=0$, $m_{u}>0$. Let also $M(q) \succ 0$.

${ }^{2}\left(x_{1} x_{2} \ldots x_{n}\right) \succcurlyeq 0$ if the first nonzero entry $x_{j}>0$ or all entries are zero. 
(i) The LCP (9) has a unique solution for any $w_{u}(q, \dot{q}, t)$ if and only if the constraint functions $h_{\mathrm{n}, u, i}(q)$ are independent ${ }^{3}(\Rightarrow m \leq n)$.

(ii) If $\lambda_{\mathrm{n}, u, 1}$ and $\lambda_{\mathrm{n}, u, 2}$ are any two solutions of the LCP in (9), then $\nabla h_{\mathrm{n}, u}^{T}(q) \lambda_{\mathrm{n}, u, 1}=$ $\nabla h_{\mathrm{n}, u}^{T}(q) \lambda_{\mathrm{n}, u, 2}$ and $w_{u}(q, \dot{q}, t)^{T} \lambda_{\mathrm{n}, u, 1}=w_{u}(q, \dot{q}, t)^{T} \lambda_{\mathrm{n}, u, 2}$. If the LCP in (9) has at least one solution at $t$, and given unique $(q, \dot{q})$, then $\ddot{q}$ is unique.

(iii) The LCP in (9) is solvable if for any $z \in \mathbb{R}^{m}$ in the set of solutions of the homogeneous $L C P$ such that $0 \leq z \perp A_{n u}(q) z \geq 0$, we have $z^{T} w_{u}(q, \dot{q}, t) \geq 0$.

(iv) The implication $\lambda_{\mathrm{n}, u} \geq 0, \nabla h_{\mathrm{n}, u}(q) \lambda_{\mathrm{n}, u}=0 \Rightarrow \lambda_{\mathrm{n}, u}^{T} \frac{d}{d t}\left(\nabla h_{\mathrm{n}, u}(q)^{T}\right) \dot{q} \geq 0$ holds if and only if the LCP in (9) is solvable.

(v) If $\frac{d}{d t}\left(\nabla h_{\mathrm{n}, u}(q)^{T}\right) \dot{q} \in \operatorname{Im}\left(A_{n u}(q)\right)$ then the LCP in (9) is solvable.

Let now $M(q) \succeq 0$ and the gap functions $h_{\mathrm{n}, u, i}(q), 1 \leq i \leq m_{u}$ satisfy the MangasarianFromovitz constraint qualification [19].

(vi) The MLCP in (8) is solvable if $T_{\Phi_{u}}(q) \cap \operatorname{Ker}(M(q))=\{0\}$, where $T_{\Phi_{u}}(q)=\left\{v \in \mathbb{R}^{n} \mid\right.$ $\left.\nabla h_{\mathrm{n}, u}(q)^{T} v \geq 0\right\}$ and $\Phi_{u}=\left\{q \in \mathbb{R}^{n} \mid h_{\mathrm{n}, u}(q) \geq 0\right\}$.

Proof Item (i) follows from the symmetry of $A_{n u}(q)$ and the important fact that $\operatorname{Ker}\left(A_{n u}(q)\right)$ $=\operatorname{Ker}\left(\nabla h_{\mathrm{n}, u}(q)\right)$ since the symmetric positive semidefinite matrix $A_{n u}(q)$ is a P-matrix if and only if it is invertible. Item (ii) is a direct application of Theorem 1 in Appendix B and implies that the mere existence of a solution to the LCP in (9) assures that the acceleration is unique, independently of $n, m$ and the constraints Jacobian matrix rank. Item (iii) follows from Theorem 2 in Appendix $\mathrm{C}$ since $A_{n u}(q)$ is positive semidefinite and hence copositive. Item (iv) is stated in [49, pp. 211-212] without proof, and we give the details of how to prove (iv) and (v) for completeness. For the "only if" part in item (iv), we notice that the condition $\left(\lambda_{\mathrm{n}, u} \geq 0, \nabla h_{\mathrm{n}, u}(q) \lambda_{\mathrm{n}, u}=0\right)$ is equivalent to $\left(\lambda_{\mathrm{n}, u} \geq 0, \lambda_{\mathrm{n}, u} \in \operatorname{Ker}\left(A_{n u}(q)\right)\right)$, which is in turn equivalent to $\lambda_{\mathrm{n}, u}$ being a solution of the homogeneous $\operatorname{LCP}\left(A_{n u}(q), 0\right)$. Next, we have the inequality $\lambda_{\mathrm{n}, u}^{T}\left(-\nabla h_{\mathrm{n}, u}(q)^{T} M(q)^{-1} F(q, \dot{q}, t)+\frac{d}{d t}\left(\nabla h_{\mathrm{n}, u}(q)^{T}\right) \dot{q}\right) \geq 0$, the first term being zero and the second nonnegative by hypothesis. Hence, the conditions for item (iii) hold, and the $\operatorname{LCP}\left(A_{n u}(q), w_{u}(q, \dot{q}, t)\right)$ is solvable. Conversely, if $\operatorname{LCP}\left(A_{n u}(q), w_{u}(q, \dot{q}, t)\right)$ is solvable, then there exists a feasible element $z$ such that $A_{n u}(q) z+w_{u}(q, \dot{q}, t) \geq 0$. Let $\lambda_{\mathrm{n}, u}$ be any element such that $\lambda_{\mathrm{n}, u} \geq 0, \nabla h_{\mathrm{n}, u}(q) \lambda_{\mathrm{n}, u}=0$. Then we have the inequality $\lambda_{\mathrm{n}, u}^{T}\left(A_{n u} z+w_{u}\right) \geq 0$. From the structures of $w_{u}(q, \dot{q}, t)$ and $A_{n u}(q)$ we deduce that $\lambda_{\mathrm{n}, u}^{T} \frac{d}{d t}\left(\nabla h_{\mathrm{n}, u}(q)^{T}\right) \dot{q} \geq 0$. Thus, the implication in (iv) holds. Item (v) gives sufficient conditions for the hypothesis of (iv) to hold. Any element in the range of $A_{n u}(q)$ is orthogonal to the nullspace of $A_{n u}(q)$ (which is the same as $\operatorname{Ker}\left(\nabla h_{\mathrm{n}, u}(q)\right)$ ). Hence, if $\frac{d}{d t}\left(\nabla h_{\mathrm{n}, u}(q)^{T}\right) \dot{q} \in \operatorname{Im}\left(A_{n u}(q)\right)$, then for all nonnegative $\lambda_{\mathrm{n}, u}$ in the nullspace of $A_{n u}(q)$, we have $\lambda_{\mathrm{n}, u}^{T} \frac{d}{d t}\left(\nabla h_{\mathrm{n}, u}(q)^{T}\right) \dot{q}=0$, so the hypothesis of (iv) holds. The proof of item (vi) relies on the results in [3]; see [11].

It is interesting to compare Proposition 3(vi) and (6): the null space is replaced by the tangent cone. In [9], item (iv) is stated as $\frac{d}{d t}\left(\nabla h_{\mathrm{n}, u}(q)\right) \dot{q}=0$; however, due to the structure of $w_{u}(q, \dot{q}, t)$, it may be replaced by this inequality as noted in [49, pp. 211-212]. It is worth noting that in item (iv) the force $F(q, \dot{q}, t)$ plays no role. Let us note that if $w_{u}(q, \dot{q}, t) \geq 0$, then the LCP in (9) is solvable since $\lambda_{\mathrm{n}, u}=0$ is a solution (possibly not the only one). This is however a rather stringent requirement. Corollary 3.8.12 in [16] may also be used in our

\footnotetext{
${ }^{3}$ That is, the gradients $\nabla h_{\mathrm{n}, u, i}(q)$ are $m$ linearly independent vectors of $\mathbb{R}^{n}$; equivalently, $\nabla h_{\mathrm{n}, u}(q)$ has rank $m$.
} 
context. Indeed, given that $A_{n u}(q)=A_{n u}(q)^{T} \succeq 0$, the LCP in (9) is equivalent to the convex quadratic program

$$
\lambda_{\mathrm{n}, u}=\operatorname{argmin}_{z \geq 0}\left(\frac{1}{2} z^{T} A_{n u}(q) z+w_{u}(q, \dot{q}, t)^{T} z\right) .
$$

This QP is solvable if and only if it is bounded from below on the set $\{z \mid z \geq 0, z \in$ $\left.\operatorname{Ker}\left(A_{n u}(q)\right)\right\}$, hence retrieving the condition of item (iv). We shall see that when friction is considered, the positivity of the QP matrix may be kept but, however, the symmetry is usually lost.

The uniqueness of $\ddot{q}$ may be deduced following another path that requires some convex analysis and variational inequalities theory (whereas proving Proposition 3 requires tools from complementarity theory [16]). The complementarity conditions in (8) are equivalently rewritten as the inclusion in a normal cone $\lambda_{\mathrm{n}, u} \in-N_{\mathbb{R}_{+}^{m_{u}}}\left(\nabla h_{\mathrm{n}, u}(q)^{T} \ddot{q}+\frac{d}{d t}\left(\nabla h_{\mathrm{n}, u}(q)^{T}\right) \dot{q}\right)$. Then using the chain rule of convex analysis (see, e.g., Proposition A.2 in [12]), it follows that

$$
\nabla h_{\mathrm{n}, u}(q) \lambda_{\mathrm{n}, u} \in-N_{K(q, \dot{q})}(\ddot{q}),
$$

where

$$
K(q, \dot{q})=\left\{x \in \mathbb{R}^{n} \mid \nabla h_{\mathrm{n}, u}(q)^{T} x+\frac{d}{d t}\left(\nabla h_{\mathrm{n}, u}(q)^{T}\right) \dot{q} \geq 0\right\}
$$

is a convex polyhedral. We suppose here that $K(q, \dot{q})$ is nonempty since otherwise the problem we are dealing with is simply meaningless. This translates into the classical constraint qualification for the chain rule [51, Theorem 23.9].

Assumption $1 \operatorname{Im}\left(\nabla h_{\mathrm{n}, u}(q)^{T}\right)$ contains a point in $\mathbb{R}_{+}^{m_{u}}-\frac{d}{d t}\left(\nabla h_{\mathrm{n}, u}(q)^{T}\right) \dot{q}$.

Therefore, the MLCP (8) is equivalently rewritten as the inclusion

$$
M(q) \ddot{q}+F(q, \dot{q}, t) \in-N_{K(q, \dot{q})}(\ddot{q}) .
$$

Proposition 4 Let $M(q) \succ 0, m$ and $n$ be arbitrary integers, and Assumption 1 hold. The inclusion in (14) has a unique solution $\ddot{q}$.

The proof follows from Theorem 6 in [3] since $M(q) \succ 0$. Consequently, the MLCP (8) with unknown $\ddot{q}$ also is well posed. This in fact was proved by Moreau [43, 44] using another reasoning. The case where $M(q)$ has low rank $r<n$ may be tackled via the tools in [3]; however, this is outside the scope of this article; see [11]. It is nevertheless worth noting that the formulations in (14) or (4) do not a priori rely on the assumption that $M(q) \succ 0$, and this will be the case of all their extensions in the sequel. The existence and uniqueness of the acceleration for given position and velocity is closely linked to Gauss' principle of mechanics (which is consequently extended to systems with unilateral constraints). Indeed, since $M(q)$ is symmetric and at least positive semi definite, (14) is equivalent to (compare with (5))

$$
\begin{aligned}
\ddot{q} & =\operatorname{argmin}_{z \in K(q, \dot{q})} \frac{1}{2} z^{T} M(q) z+z^{T} F(q, \dot{q}, t) \\
& =\operatorname{proj}_{M(q)}\left[K(q, \dot{q}) ;-M(q)^{-1} F(q, \dot{q}, t)\right] \quad(\text { in case } M(q) \succ 0) .
\end{aligned}
$$


The existence and uniqueness of $\ddot{q}$ in Proposition 3(ii) is clearly sufficient only since it relies on the existence of a solution $\lambda_{\mathrm{n}, u}$ of the contact LCP, whereas the acceleration existence relies on the existence of the generalized force $F_{\mathrm{n}, u}(q) \triangleq \nabla h_{\mathrm{n}, u}(q) \lambda_{\mathrm{n}, u}$. Further quadratic programs obtained for the unilaterally constrained case may be found in $[9, \S 4.4 .1]$, using Dorn's duality. They summarize various results (including (11) and (15)), some of which were obtained by Moreau [43, 44] and Lötstedt [38]. It is worth noting that (14) is written in a more general setting in [22, Eq. (8.11)] such that $K(q, \dot{q})=T_{T_{\Phi_{u}(q)}}(\dot{q})$. Here we deal with the case where $h_{\mathrm{n}, u}(q)=0$ and $\nabla h_{\mathrm{n}, u}(q)^{T} \dot{q}=0$, that is, $q \in \operatorname{bd}\left(\Phi_{u}\right)$ and $\dot{q} \in \operatorname{bd}\left(T_{\Phi_{u}}(q)\right)$ since it is clear that if $h_{\mathrm{n}, u}(q)=0$ and $\nabla h_{\mathrm{n}, u}(q)^{T} \dot{q}>0$, or if $h_{\mathrm{n}, u}(q)>0$, then $\ddot{q}$ is unsigned.

\subsection{Unilaterally/bilaterally constrained systems}

We now consider the case where both bilateral and unilateral constraints are involved. Surprizingly enough, it is only recently that the analysis of this case has received attention $[9,11]$, though it may represent the most common case in practice [50]. Let us write the bilateral constraints on the acceleration level. The MLCP in (8) is augmented as follows:

$$
\left\{\begin{array}{l}
\text { (a) } M(q) \ddot{q}+F(q, \dot{q}, t)=\nabla h_{\mathrm{n}, b}(q) \lambda_{\mathrm{n}, b}+\nabla h_{\mathrm{n}, u}(q) \lambda_{\mathrm{n}, u}, \\
\text { (b) } 0 \leq \lambda_{\mathrm{n}, u} \perp \nabla h_{\mathrm{n}, u}(q)^{T} \ddot{q}+\frac{d}{d t}\left(\nabla h_{\mathrm{n}, u}(q)^{T}\right) \dot{q} \geq 0, \\
\text { (c) } \nabla h_{\mathrm{n}, b}(q)^{T} \ddot{q}+\frac{d}{d t}\left(\nabla h_{\mathrm{n}, b}(q)^{T}\right) \dot{q}=0 .
\end{array}\right.
$$

In the following, we are going to follow different paths for the analysis of this MLCP.

\subsubsection{Analysis of acceleration inclusions}

Existence and uniqueness of $\ddot{\boldsymbol{q}}$ via a first inclusion Let us try to mimic the developments of Sect. 2.2, in particular, the steps that lead to inclusion (14). The MLCP in (16) is a mixture of equality and complementarity constraints. Let us make the following assumption.

Assumption $2 M(q) \succ 0$, and the $m_{b} \times m_{b}$ matrix $A_{n b}(q)$ is positive definite for all $q \in \mathbb{R}^{n}$ $\left(\Leftrightarrow \nabla h_{\mathrm{n}, b}(q)\right.$ has rank $\left.m_{b}\right)$.

Under this assumption, $\ddot{q}$ can be eliminated from (16)(a) and its expression injected in (16)(c), yielding

$$
\begin{aligned}
& A_{\mathrm{nb}}(q) \lambda_{\mathrm{n}, b}+\underbrace{\nabla h_{\mathrm{n}, b}(q)^{T} M(q)^{-1} \nabla h_{\mathrm{n}, u}(q)}_{A_{\mathrm{nbnu}}(q)} \lambda_{\mathrm{n}, u}-\nabla h_{\mathrm{n}, b}(q)^{T} M(q)^{-1} F(q, \dot{q}, t) \\
& \quad+\frac{d}{d t}\left(\nabla h_{\mathrm{n}, b}(q)^{T}\right) \dot{q}=0 .
\end{aligned}
$$

We may use the latter equality to express $\lambda_{\mathrm{n}, b}$ as a function of $\lambda_{\mathrm{n}, u}$ (the couplings between both sets of multipliers come from the matrix $A_{\mathrm{nbnu}}(q) \triangleq \nabla h_{\mathrm{n}, b}(q)^{T} M(q)^{-1} \nabla h_{\mathrm{n}, u}(q)$; this shows why jumps in $\lambda_{\mathrm{n}, u}$ may induce jumps in $\lambda_{\mathrm{n}, b}$ ). Inserting this expression into the dy- 
namics, we obtain:

$$
\begin{aligned}
& M(q) \ddot{q}+P(q) F(q, \dot{q}, t)+\nabla h_{\mathrm{n}, b}(q)\left(\nabla h_{\mathrm{n}, b}(q)^{T} M(q)^{-1} \nabla h_{\mathrm{n}, b}(q)\right)^{-1} \frac{d}{d t}\left(\nabla h_{\mathrm{n}, b}(q)^{T}\right) \dot{q} \\
& \quad=P(q) \nabla h_{\mathrm{n}, u}(q) \lambda_{\mathrm{n}, u}, \\
& 0 \leq \lambda_{\mathrm{n}, u} \perp \nabla h_{\mathrm{n}, u}(q)^{T} \ddot{q}+\frac{d}{d t}\left(\nabla h_{\mathrm{n}, u}(q)^{T}\right) \dot{q} \geq 0,
\end{aligned}
$$

with

$$
P(q) \triangleq I-\nabla h_{\mathrm{n}, b}(q) A_{n b}(q)^{-1} \nabla h_{\mathrm{n}, b}(q)^{T} M(q)^{-1} .
$$

This is equivalent to an inclusion that extends (14):

$$
M(q) \ddot{q}+P(q) F(q, \dot{q}, t)+\nabla h_{\mathrm{n}, b}(q) A_{n b}(q)^{-1} \frac{d}{d t}\left(\nabla h_{\mathrm{n}, b}(q)^{T}\right) \dot{q} \in-P(q) N_{K(q, \dot{q})}(\ddot{q}) .
$$

To obtain (20), we used (12). Three facts about $P(q) \in \mathbb{R}^{n \times n}$ are: $\nabla h_{\mathrm{n}, b}(q)^{T} M(q)^{-1} P(q)$ $=0, M(q)^{-1} P(q) \in \mathbb{R}^{n \times n}$ is symmetric positive semidefinite with rank $r=n-m_{b}$, and $\operatorname{rank}(P(q)) \leq n-1$. The first fact is obvious from (19), the second fact follows from Lemmas 2 and 3 in [9], and the third fact is a consequence of [6, Fact 3.8.6]. The first fact may be used to prove that the submanifold $\left\{(x, y) \in \mathbb{R}^{n} \times \mathbb{R}^{n} \mid h_{\mathrm{n}, b}(x)=0, \nabla h_{\mathrm{n}, b}(q)^{T} y=0\right\}$ is invariant under the dynamics in (20), which means that the multiplier $\lambda_{\mathrm{n}, b}$ plays the role of a contact force that maintains the system on the bilateral constraint. We deduced the uniqueness of $\ddot{q}$ from (14). However, (20) is more complex because of the presence of the singular matrix $P(q)$, which is an idempotent matrix. $P(q)$ is not symmetric, and hence it is not a projector according to [6, Definition 3.1.1], but it may be named a projector according to $[33, \S 5.8]$ (onto $\operatorname{Im}(P(q))$ along $\operatorname{Ker}(P(q))$ ). Let us rewrite (20) more compactly as

$$
\ddot{q}+D(q, \dot{q}, t) \in-M(q)^{-1} P(q) N_{K(q, \dot{q})}(\ddot{q})
$$

with an obvious definition of $D(q, \dot{q}, t)$. Since $M(q)^{-1} P(q)=\left(M(q)^{-1} P(q)\right)^{T} \succeq 0$, there exists a unitary matrix $S(q)$ such that $S(q) M(q)^{-1} P(q) S(q)^{T}=\left(\begin{array}{cc}P_{r}(q) & 0 \\ 0 & 0\end{array}\right)$ with $P_{r}(q)=$ $P_{r}(q)^{T} \succ 0$ of dimension $\left(n-m_{b}\right) \times\left(n-m_{b}\right)$. Let $z \triangleq S(q) \ddot{q}$. Then $(21)$ is rewritten equivalently as

$$
z+S(q) D(q, \dot{q}, t) \in-\left(\begin{array}{cc}
P_{r}(q) & 0 \\
0 & 0
\end{array}\right) S(q) N_{K(q, \dot{q})}\left(S(q)^{T} z\right)
$$

The chain rule of convex analysis [51, Theorem 23.9] allows us to state that $S(q) N_{K(q, \dot{q})} \times$ $\left(S(q)^{T} z\right)=N_{\Phi(q, \dot{q})}(z)$ with $\Phi(q, \dot{q})=\left\{z \in \mathbb{R}^{n} \mid S(q)^{T} z \in K(q, \dot{q})\right\}=\left(S(q)^{T}\right)^{-1}(K(q, \dot{q}))$. For $x \in \mathbb{R}^{n}$, we denote by $x_{r}$ the vector made of its first $r$ entries and by $x_{n-r}$ the vector made of its last $n-r$ entries. From (22) we infer that

$$
z_{n-r}=-(S(q) D(q, \dot{q}))_{n-r} \Leftrightarrow S_{n-r}(q) \ddot{q}=-S_{n-r}(q) D(q, \dot{q}, t),
$$

where $S_{n-r}(q) \in \mathbb{R}^{(n-r) \times n}$ is the matrix made of the last $n-r$ rows of $S(q)$, whereas $S_{r}(q) \in$ $\mathbb{R}^{(r) \times n}$ is the matrix made of the first $r$ rows of $S(q)$. We make the following assumption.

Assumption 3 The set $\Phi(q, \dot{q})=\left\{z \in \mathbb{R}^{n}\left|z_{n-r}=-(S(q) D(q, \dot{q}, t))_{n-r}\right| S(q)^{T} z \in K(q, \dot{q})\right\}$ is nonempty. 
It is worth noting that Assumption 3 imposes some constraint on the term $D(q, \dot{q}, t)$ defined from (21). Let us denote $\Phi(q, \dot{q})$ as $\Phi_{r}(q, \dot{q})$ since the free variable is $z_{r}$, that is,

$$
\begin{aligned}
\Phi_{r}(q, \dot{q})= & \left\{z_{r} \in \mathbb{R}^{r} \mid\left(\nabla h_{\mathrm{n}, u}(q)^{T} S(q)^{T}\right)_{r} z_{r}+\left(\nabla h_{\mathrm{n}, u}(q)^{T} S(q)^{T}\right)_{n-r}(-S(q) D(q, \dot{q}, t))_{n-r}\right. \\
& \left.+\frac{d}{d t}\left(\nabla h_{\mathrm{n}, u}(q)^{T}\right) \dot{q} \geq 0\right\} .
\end{aligned}
$$

In fact, Assumption 3 states that $\Phi_{r}(q, \dot{q})$ is nonempty. We can rewrite $N_{\Phi(q, \dot{q})}(z)$ equivalently as

$$
\left(\begin{array}{c}
\partial_{z_{r}} \Psi_{\Phi(q, \dot{q})}(z) \\
\partial_{z_{n-r}} \Psi_{\Phi(q, \dot{q})}(z)
\end{array}\right)=\left(\begin{array}{c}
\partial_{z_{r}} \Psi_{\Phi_{r}(q, \dot{q})}\left(z_{r}\right) \\
\partial_{z_{n-r}} \Psi_{\Phi(q, \dot{q})}(z)
\end{array}\right),
$$

where $\Psi_{\Phi_{r}(q, \dot{q})}\left(z_{r}\right)=\psi_{\mathbb{R}_{m_{u}}^{+}} \circ\left[\left(\nabla h_{\mathrm{n}, u}(q)^{T} S(q)^{T}\right)_{r} z_{r}+w_{r}(q, \dot{q})\right]$ with

$$
w_{r}(q, \dot{q}) \triangleq\left(\nabla h_{\mathrm{n}, u}(q)^{T} S(q)^{T}\right)_{n-r}(-S(q) F(q, \dot{q}, t))_{n-r}+\frac{d}{d t}\left(\nabla h_{\mathrm{n}, u}(q)^{T}\right) \dot{q} .
$$

Thus, from (22) and (23) we get

$$
z_{r}+(S(q) D(q, \dot{q}, t))_{r} \in-P_{r}(q) N_{\Phi_{r}(q, \dot{q})}\left(z_{r}\right) .
$$

Since $P_{r}(q)=P_{r}(q)^{T} \succ 0$, so is its inverse, and we can apply again Theorem 6 in [3] to conclude about the existence and uniqueness of a solution $z_{r}$ to (25). Since $\ddot{q}=S(q)^{-1} z$, we thus proved the following.

Proposition 5 Let $F(q, \dot{q}, t)$ be given. Suppose that Assumptions 1, 2, and 3 hold. The inclusion in (21) has a unique solution $z$, and the MLCP in (16) has a unique solution $\ddot{q}$.

Using (16)(a), we conclude that $\nabla h_{\mathrm{n}, b}(q) \lambda_{\mathrm{n}, b}+\nabla h_{\mathrm{n}, u}(q) \lambda_{\mathrm{n}, u}$ is unique as well. The uniqueness of the multipliers holds under an additional rank assumption on the gradients. Notice that using (25) we obtain:

$$
\begin{aligned}
& P_{r}(q)^{-1} z_{r}+P_{r}(q)^{-1}(S(q) D(q, \dot{q}, t))_{r} \in-N_{\Phi_{r}(q, \dot{q})}\left(z_{r}\right) \\
& \Leftrightarrow \quad z_{r}=\operatorname{proj}_{P_{r}(q)^{-1}}\left[\Phi_{r}(q, \dot{q}) ;(S(q) D(q, \dot{q}, t))_{r}\right] \\
& \Leftrightarrow \quad \text { Find } z_{r} \in \Phi_{r}(q, \dot{q}):\left\langle P_{r}(q)^{-1} z_{r}+P_{r}(q)^{-1}(S(q) D(q, \dot{q}, t))_{r}, v-z_{r}\right\rangle \geq 0 \\
& \quad \text { for all } v \in \Phi_{r}(q, \dot{q}),
\end{aligned}
$$

where the last formalism is a variational inequality of the first kind. Gauss' principle applies to systems subject to unilateral and bilateral constraints (as shown in [9] through various quadratic problems). It is worth noting that a direct interpretation of the inclusion in (21) as in (26) is not possible due to the singular matrix $P(q)$.

Existence and uniqueness of $\ddot{\boldsymbol{q}}$ via a second inclusion As we have seen, under Assumption 1, using (16)(b), we have $\nabla h_{\mathrm{n}, u}(q) \lambda_{\mathrm{n}, u} \in-N_{K(q, \dot{q})}(\ddot{q})$. Similarly, we have $\nabla h_{\mathrm{n}, b}(q) \lambda_{\mathrm{n}, b} \in-N_{K_{b}(q, \dot{q})}(\ddot{q})$ with $K_{b}(q, \dot{q})=\left\{z \in \mathbb{R}^{n} \mid \nabla h_{\mathrm{n}, b}(q)^{T} z+\frac{d}{d t}\left(\nabla h_{\mathrm{n}, b}(q)^{T}\right) \dot{q}=0\right\},{ }^{4}$

\footnotetext{
${ }^{4}$ Here the normal cone to $K_{b}(q, \dot{q})$ is simply generated by the gradients $\nabla h_{\mathrm{n}, b, i}(q), 1 \leq i \leq m_{b}$.
} 
the assumption being here that $K_{b}(q, \dot{q})$ is nonempty: this inclusion enforces the bilateral constraints.

Assumption 4 The set $K(q, \dot{q}) \cap K_{b}(q, \dot{q})$ is nonempty.

Then we have the following [11] (compare with (5), (15), (26)):

Proposition 6 Let Assumptions 1 and 4 hold. The MLCP in (16) is equivalent to

$$
\begin{gathered}
M(q) \ddot{q}+F(q, \dot{q}, t) \in-N_{K(q, \dot{q}) \cap K_{b}(q, \dot{q})}(\ddot{q}) \\
\Leftrightarrow \quad \text { Find } \ddot{q} \in K(q, \dot{q}) \cap K_{b}(q, \dot{q}):\langle M(q) \ddot{q}+F(q, \dot{q}, t), v-\ddot{q}\rangle \geq 0 \\
\quad \text { for all } v \in K(q, \dot{q}) \cap K_{b}(q, \dot{q}) \\
\Leftrightarrow \quad \ddot{q}=\operatorname{argmin}_{z \in K(q, \dot{q}) \cap K_{b}(q, \dot{q})} \frac{1}{2} z^{T} M(q) z+z^{T} F(q, \dot{q}, t) .
\end{gathered}
$$

Notice that no assumption on the rank of $M(q)$ has been made to get (27). In order to get the right-hand side of the inclusion in (27), we used [51, Theorem 23.8] about the subdifferential of the sum of convex functions and the fact that the functions we are dealing with are polyhedral.

Corollary 1 Let $F(q, \dot{q}, t)$ be given. Suppose that $M(q) \succ 0$ and that Assumptions 1 and 4 hold. Then $\ddot{q}$ is given by

$$
\ddot{q}=\operatorname{proj}_{M(q)}\left[K(q, \dot{q}) \cap K_{b}(q, \dot{q}) ;-M(q)^{-1} F(q, \dot{q}, t)\right] .
$$

In fact, Assumption 4 implies that Assumption 1 holds. Let us notice that $K(q, \dot{q}) \cap$ $K_{b}(q, \dot{q})$ is convex; hence, the projection is indeed uniquely defined. The inclusions in (27) and (25) are not equivalent because no assumption on the rank of $M(q)$ has been made to get (27), contrary to (25) (indeed, Assumption 2 holds only if $M(q) \succ 0$ ). Again, we may analyze (27) with $M(q) \succeq 0$ only, relying on results in [3] and [11]. This may be particularly interesting when bilateral constraints are present. Indeed, singular mass matrices are often due to the use of redundant generalized coordinates together with bilateral holonomic constraints $[26,59,60]$. We may use [3, Corollary 4] to cope with (27) with a low-rank $M(q)$, but this would bring us too far away from the main topic of this paper. Some results in this direction may be found in [11].

Existence and uniqueness of $\ddot{q}$ and $\lambda_{n, b}$ via a third inclusion Let us investigate again how one may dispense with Assumption 2 (as well as with the underlying assumption that $M(q) \succ 0)$. In (27) and (25), the multiplier $\lambda_{\mathrm{n}, b}$ does not appear explicitly because it has been eliminated in the process used to obtain these two inclusions. Let us investigate a path that yields a direct extension of the KKT system (4). Using (12) with $K(q, \dot{q})$ in (13), it is possible to rewrite (16) as follows:

$$
\left(\begin{array}{cc}
M(q) & -\nabla h_{\mathrm{n}, b}(q) \\
\nabla h_{\mathrm{n}, b}(q)^{T} & 0
\end{array}\right)\left(\begin{array}{c}
\ddot{q} \\
\lambda_{\mathrm{n}, b}
\end{array}\right)+\left(\begin{array}{c}
F(q, \dot{q}, t) \\
\frac{d}{d t}\left(\nabla h_{\mathrm{n}, b}(q)^{T}\right) \dot{q}
\end{array}\right) \in\left(\begin{array}{c}
-N_{K(q, \dot{q})}(\ddot{q}) \\
0
\end{array}\right) .
$$

Inclusion (29) represents the merging between (4) and (14). As noticed in Sect. 2.1, the DAE matrix $M_{b}(q)$ in the left-hand side of (29) is positive semidefinite and nonsymmetric. Let us 
denote the second term in the left-hand side as $F_{b}(q, \dot{q}, t)$. We have that $\{0\}=N_{\mathbb{R}^{m}}\left(\lambda_{\mathrm{n}, b}\right)$. Let us define $z \triangleq\left(\begin{array}{c}\ddot{q} \\ \lambda_{\mathrm{n}, b}\end{array}\right)$ and $f_{(q, \dot{q})}(z)=\Psi_{K(q, \dot{q})}(\ddot{q})+\Psi_{\mathbb{R}^{m} b}\left(\lambda_{\mathrm{n}, b}\right)$. Using Assumption 1, we may therefore rewrite equivalently (compare with (14) and (27)):

$$
\begin{aligned}
& M_{b}(q) z+F_{b}(q, \dot{q}, t) \in-\partial f_{(q, \dot{q})}(z) \\
& \Leftrightarrow \quad \text { Find } z \in \mathbb{R}^{n+m_{b}}:\left\langle M_{b}(q) z+F_{b}(q, \dot{q}, t), v-z\right\rangle+f_{(q, \dot{q})}(v)-f_{(q, \dot{q})}(z) \geq 0, \\
& \quad \text { for all } v \in \mathbb{R}^{n+m_{b}},
\end{aligned}
$$

which is a variational inequality of the second kind.

It is worth noting that despite $M_{b}(q) \in \mathbb{R}^{\left(n+m_{b}\right) \times\left(n+m_{b}\right)}$ is positive semidefinite, it may have full rank due to its skew-symmetric part. Using Appendix E, item (iii'), it follows that $M_{b}(q)$ is invertible if and only if $\operatorname{Ker}(M(q)) \cap \operatorname{Ker}\left(\nabla h_{\mathrm{n}, b}(q)^{T}\right)=\{0\}$ and $\operatorname{rank}\left(\nabla h_{\mathrm{n}, b}(q)^{T}\right)=m_{b}$. We may then conclude about the well-posedness of the system $M_{b}(q) z+F_{b}(q, \dot{q}, t)=0$.

However, direct conclusions on the well-posedness of (30) cannot be drawn even if $M_{b}(q)$ satisfies the conditions recalled in Appendix E since the well-posedness of inclusions like (30) relies on positivity-like properties ( $P$-matrices, copositive matrices, positive definiteness) and not merely on rank assumptions. This makes a strong difference between problems involving unilateral constraints and those with bilateral constraints only.

We note that the criteria presented in $[3,11]$ rely on semicomplementarity problems that use recession functions and cones. Introducing such mathematical tools is not possible here for the sake of briefness. Nevertheless, the crucial subspace that plays a role in the various criteria proposed in [3] is $\operatorname{Ker}\left(M_{b}(q)+M_{b}(q)^{T}\right)=\left\{z \in \mathbb{R}^{n+m_{b}} \mid M(q) \ddot{q}=0\right\}$ (see [3, Corollary 3], which applies to nonsymmetric positive semidefinite matrices).

Remark 1 The other (mechanically equivalent) option in (4) or (29) is to replace $-\nabla h_{\mathrm{n}, b}(q)$ with $\nabla h_{\mathrm{n}, b}(q)$. As pointed out in Sect. 2.1, $M_{b}(q)$ is then symmetric, however, never positive semidefinite (a necessary condition for its positive semidefiniteness is that $-\nabla h_{\mathrm{n}, b}(q)^{T} M(q)^{-1} \nabla h_{\mathrm{n}, b}(q)$ is positive semidefinite, which is possible only if $\nabla h_{\mathrm{n}, b}(q)=$ $0)$. The criteria based on positiveness and its variants cannot be used.

The solvability Theorem 2.1 in [40] applies to (29) only if $\frac{d}{d t}\left(\nabla h_{\mathrm{n}, b}(q)^{T}\right) \dot{q} \geq 0$ (componentwise nonnegativity), which is a rather restrictive case, which holds in practice for all velocities only if $\frac{d}{d t}\left(\nabla h_{\mathrm{n}, b}(q)^{T}\right)=0$ (therefore, it has practical interest for the static case where $\dot{q}=0$ ). This in turn implies that the index 1 formalism (constraints written on the acceleration level) and the index 2 formalism (constraints written on the velocity level) possess the same canonical form.

Conclusions Three inclusions have been constructed that extend the KKT system (4) and inclusion (14) when both bilateral and unilateral constraints are present: (25), (27), and (30). They rely on different assumptions, which are used to transform the MLCP in (16). There is a significant discrepancy between Assumptions 1, 3, and 4 (which are necessary to secure that the problems make sense, i.e., we do not consider a system with an empty admissible space) and Assumption 2. Assumption 2 (with the prerequisite that $M(q) \succ 0$ ) allows us to calculate $\lambda_{\mathrm{n}, b}$ and inject its expression into the dynamics in order to obtain a projected dynamics on the bilateral constraints. On the contrary, in (27), the bilateral constraints are enforced directly in the normal cone. Finally, (29) uses only Assumption 1. Both (27) and (29) hold with $M(q) \succeq 0$ and possibly redundant constraints. 


\subsubsection{Analysis of contact LCP with a bilateral distortion}

Another way is chosen in [9]. Instead of augmenting the unilateral constraints set, one uses as above the second equality in (16) to get an expression of $\lambda_{\mathrm{n}, b}$ as a function of $\lambda_{\mathrm{n}, u}$ and inserts it into the dynamics (the first line of (16)). Let Assumption 2 hold. Then the constrained LCP is obtained in the same way as (9):

$$
0 \leq \lambda_{\mathrm{n}, u} \perp A_{c}(q) \lambda_{\mathrm{n}, u}+w_{c}(q, \dot{q}, t) \geq 0,
$$

where the new Delassus matrix is

$$
A_{c}(q) \triangleq \nabla h_{\mathrm{n}, u}(q)^{T} M_{c}(q)^{-1} \nabla h_{\mathrm{n}, u}(q)
$$

with $M_{c}(q)^{-1} \triangleq M(q)^{-1} P(q)$ and $P(q)$ as in (19). We see that $M_{c}(q)^{-1}$ is the symmetric positive semidefinite matrix ${ }^{5}$ that appears in the right-hand side of (21): it is the inverse mass matrix with a distortion due to the bilateral holonomic constraints. Hence, $A_{c}(q)=A_{c}(q)^{T}$ is at least positive semidefinite. Finally, we have

$$
\begin{aligned}
w_{c}(q, \dot{q}, t)= & \nabla h_{\mathrm{n}, u}(q)^{T} M_{c}(q)^{-1} F_{M}(q, \dot{q}, t)+\nabla h_{\mathrm{n}, u}(q)^{T} M^{-1}(q) \frac{d}{d t}\left(\nabla h_{\mathrm{n}, b}(q)^{T}\right) \dot{q} \\
& +\frac{d}{d t}\left(\nabla h_{\mathrm{n}, u}(q)^{T}\right) \dot{q}
\end{aligned}
$$

with $F_{M}(q, \dot{q}, t) \triangleq-M(q) F(q, \dot{q}, t)$. Clearly, the LCP in (31), (32), and (33) is the extension of the LCP in (9) and (10), where $M(q)^{-1}$ is replaced by $M_{c}(q)^{-1}$ and $w_{u}(q, \dot{q}, t)$ by $w_{c}(q, \dot{q}, t)$. A proposition quite in the same spirit as Proposition 3 may be stated, which concatenates Proposition 6, Corollary 1, and Lemma 4 in [9] and results from [11].

Proposition 7 (Frictionless, unilateral/bilateral constraints) Let Assumption 2 hold. Then:

(i) The LCP in (31) has a unique solution for any $w_{c}(q, \dot{q}, t)$ if and only if $A_{c}(q) \succ 0$ or, equivalently, $\left(\nabla h_{\mathrm{n}, u}(q) \nabla h_{\mathrm{n}, b}(q)\right)$ is of full rank.

(ii) Let $\lambda_{\mathrm{n}, u, 1}$ and $\lambda_{\mathrm{n}, u, 2}$ be two solutions of the contact LCP in (31). Then $\nabla h_{\mathrm{n}, u}(q)\left(\lambda_{\mathrm{n}, u, 1}-\right.$ $\left.\lambda_{\mathrm{n}, u, 2}\right) \in \operatorname{Ker}\left(M_{c}(q)^{-1}\right)$, and $\left(\lambda_{\mathrm{n}, u, 1}^{T}-\lambda_{\mathrm{n}, u, 2}^{T}\right) w_{c}(q, \dot{q})=0$.

(iii) Let $\operatorname{Im}\left(\nabla h_{\mathrm{n}, u}(q)\right) \cap \operatorname{Im}\left(\nabla h_{\mathrm{n}, b}(q)\right)=\{0\}$ for all $q \in \mathbb{R}^{n}$. Let $\lambda_{\mathrm{n}, u, 1}$ and $\lambda_{\mathrm{n}, u, 2}$ be two solutions of the LCP in (31). Then $\nabla h_{\mathrm{n}, u}(q)\left(\lambda_{\mathrm{n}, u, 1}-\lambda_{\mathrm{n}, u, 2}\right)=0$. If the LCP in (31) is solvable, then the acceleration $\ddot{q}$ exists and is unique.

(iv) The LCP in (31) is solvable if for any $z \in \mathbb{R}^{n}$ in the set of solutions of the homogeneous $L C P$ such that $0 \leq z \perp A_{c}(q) z \geq 0$, we have $z^{T} w_{c}(q, \dot{q}, t) \geq 0$; if $\frac{d}{d t}\left(\nabla h_{\mathrm{n}, b}(q)\right)=0$, $\frac{d}{d t}\left(\nabla h_{\mathrm{n}, u}(q)\right)=0$, and $\operatorname{Im}\left(\nabla h_{\mathrm{n}, u}(q)\right) \cap \operatorname{Im}\left(\nabla h_{\mathrm{n}, b}(q)\right)=\{0\}$ for all $q \in \mathbb{R}^{n}$, then the LCP in (31) is solvable.

(v) The implication $\lambda_{\mathrm{n}, u} \geq 0, \nabla h_{\mathrm{n}, u}(q) \lambda_{\mathrm{n}, u}=0 \Rightarrow \lambda_{\mathrm{n}, u}^{T} \frac{d}{d t}\left(\nabla h_{\mathrm{n}, u}(q)^{T}\right) \dot{q} \geq 0$ holds if and only if the LCP in (31) is solvable.

(vi) If $\left(\nabla h_{\mathrm{n}, u}(q)^{T} M^{-1}(q) \frac{d}{d t}\left(\nabla h_{\mathrm{n}, b}(q)^{T}\right)+\frac{d}{d t}\left(\nabla h_{\mathrm{n}, u}(q)^{T}\right)\right) \dot{q} \in \operatorname{Im}\left(A_{c}(q)\right)$, then the LCP in (31)-(33) is solvable.

Let now $M(q) \succeq 0$, and let the constraints $h_{\mathrm{n}, u, i}(q), 1 \leq i \leq m_{u}$, satisfy the MangasarianFromovitz constraint qualification. Then:

\footnotetext{
${ }^{5}$ This is denoted as the inverse of some matrix though there is no inverse, just to mimic the case without bilateral constraints.
} 
(vii) The MLCP in (16) (equivalently, (27)) is solvable if $\operatorname{Ker}\left(\nabla h_{\mathrm{n}, b}(q)\right) \cap T_{\Phi_{u}}(q) \cap$ $\operatorname{Ker}(M(q))=\{0\}$.

Item (v) stems from similar arguments as item (iv) of Proposition 3, using the specific form of $w_{c}(q, \dot{q}, t)$ in (33), and similarly for item (vi) (these facts were not noticed in [9]). Item (iv), that is, Lemma 4 in [9], therefore states sufficient conditions only as a direct consequence of Theorem 2 in Appendix C. As in Proposition 3, both positivity and symmetry of $A_{c}(q)$ are used. It is worth noting that the independence of the constraints $h_{\mathrm{n}, u, i}(q)$ is not sufficient to assure (i) as in Proposition 3 because $M_{c}(q)^{-1}$ is not a full-rank matrix. Conditions that guarantee that $A_{c}(q) \succ 0$ are given in [9], mainly relying on the kinetic angles values. It has been noticed in another context [29, Lemma 2] that a matrix with the same structure as $A_{c}(q)$ is the Schur complement of $A_{n b}(q)$ in $\left(\begin{array}{c}\nabla h_{\mathrm{n}, b}(q)^{T} \\ \nabla h_{\mathrm{n}, u}(q)^{T}\end{array}\right) M(q)^{-1}\left(\nabla h_{\mathrm{n}, b}(q) \nabla h_{\mathrm{n}, u}(q)\right)=\left(\begin{array}{cc}A_{n b}(q) & A_{n b n u}(q) \\ A_{n b n u}(q) & A_{n u}(q)\end{array}\right)$. Hence, $A_{c}(q)=A_{n u}(q)-A_{n b n u}(q)^{T} A_{n b}(q)^{-1} A_{n b n u}(q)$ is positive definite if and only if $\left(\begin{array}{c}\nabla h_{\mathrm{n}, b}(q)^{T} \\ \nabla h_{\mathrm{n}, u}(q)^{T}\end{array}\right)$ has full row rank $\Leftrightarrow \operatorname{Ker}\left(\nabla h_{\mathrm{n}, b}(q) \nabla h_{\mathrm{n}, u}(q)\right)=\{0\}$ [29, Lemma 4] (a condition easier to check than the necessary and sufficient condition of [9, Proposition 10]). The Uniqueness of the acceleration in item (iii) holds since the right-hand side of the dynamic equation is equal to $P(q) \nabla h_{\mathrm{n}, u}(q) \lambda_{\mathrm{n}, u, 1}$.

Remark 2 Consider the solvability conditions in Propositions 3 and 7. Using Corollary 3.8.12 in [16], we have that the solvability of the $\operatorname{LCP}\left(w_{u}(q, \dot{q}, t), A_{n u}(q)\right)$ is equivalent to the boundedness from below of the quadratic function $f(z)=z^{T} w_{u}(q, \dot{q}, t)+z^{T} A_{n u}(q) z$ for all $z \geq 0$ (and the same for $w_{c}(q, \dot{q}, t)$ and $A_{c}(q)$ ). It is known that such $f(z)$ is bounded from below for all $z$ (not necessarily nonnegative) if and only if $w_{n u}(q, \dot{q}, t) \in$ $\operatorname{Im}\left(A_{n u}(q)\right)$ [8, §a.5.5]. Thus, the condition that $w_{u}(q, \dot{q}, t) \in \operatorname{Im}\left(A_{n u}(q)\right)$ may be used for solvability of the contact LCP as a sufficient condition. In view of the structures of the terms $w_{u}(q, \dot{q}, t)$ and $w_{c}(q, \dot{q}, t)$ in (10) and (33), if $\frac{d}{d t}\left(\nabla h_{\mathrm{n}, u}(q)\right) \in \operatorname{Im}\left(A_{n u}(q)\right)$ (resp. $\left.\left[\nabla h_{\mathrm{n}, u}(q)^{T} M(q)^{-1} \frac{d}{d t}\left(\nabla h_{\mathrm{n}, b}(q)\right)+\frac{d}{d t}\left(\nabla h_{\mathrm{n}, u}(q)\right)\right] \dot{q} \in \operatorname{Im}\left(A_{c}(q)\right)\right)$, then $w_{u}(q, \dot{q}, t) \in$ $\operatorname{Im}\left(A_{n u}(q)\right)\left(\operatorname{resp} . w_{c}(q, \dot{q}, t) \in \operatorname{Im}\left(A_{c}(q)\right)\right)$ if and only if $F(q, \dot{q}, t)\left(\operatorname{resp} . F_{M}(q, \dot{q}, t)\right)$ belong to $\operatorname{Im}\left(\nabla h_{\mathrm{n}, u}(q)\right)$. This is more restrictive than the conditions in Proposition 3(iv) and (v) and Proposition 7(v) and (vi).

\subsubsection{Analysis of the MLCP (16)}

Let us analyze the contact MLCP in (16) with relaxed Assumption 2.

Proposition 8 Let us consider (16). Suppose that $\frac{d}{d t}\left(\nabla h_{\mathrm{n}, b}(q)^{T}\right) \dot{q} \in \operatorname{Im}\left(\nabla h_{\mathrm{n}, b}(q)^{T}\right)$. Then the solvability of the contact LCP holds if and only if $\nabla h_{\mathrm{n}, u}(q)^{T}[z+y]+\frac{d}{d t}\left(\nabla h_{\mathrm{n}, u}(q)^{T}\right) \dot{q} \geq 0$ for some $z \in \mathbb{R}^{n}$ and $y \in \operatorname{Ker}\left(\nabla h_{\mathrm{n}, b}(q)^{T}\right)$.

Proof From (17) it follows using the assumption of the proposition that there exist $z \in \mathbb{R}^{n}$ and $y \in \operatorname{Ker}\left(\nabla h_{\mathrm{n}, b}(q)^{T}\right)$ such that $\nabla h_{\mathrm{n}, b}(q) \lambda_{\mathrm{n}, b}=-\nabla h_{\mathrm{n}, u}(q) \lambda_{\mathrm{n}, u}+F(q, \dot{q}, t)+M(q) z+$ $M(q) y$. Inserting this expression into (16) (b) and using (16) (a), we find that $\lambda_{\mathrm{n}, u}$ has to satisfy the degenerate LCP

$$
0 \leq \lambda_{\mathrm{n}, u} \perp \nabla h_{\mathrm{n}, u}(q)^{T}[z+y]+\frac{d}{d t}\left(\nabla h_{\mathrm{n}, u}(q)^{T}\right) \dot{q} \geq 0 .
$$


Clearly, this complementarity problem has a solution if and only if $\nabla h_{\mathrm{n}, u}(q)^{T}[z+y]+$ $\frac{d}{d t}\left(\nabla h_{\mathrm{n}, u}(q)^{T}\right) \dot{q} \geq 0$.

Applying Theorem 2 in Appendix $\mathrm{C}$ and noting that in the degenerate case with $M=0$, we have $\mathcal{Q}_{0}=\mathcal{Q}_{0}^{*}=\mathbb{R}_{+}^{m_{u}}$, provides a sufficient condition. It is worth noting that the assumption of the proposition is implied by Assumption 2. If (17) is seen as a linear equation with unknown $\nabla h_{\mathrm{n}, b}(q) \lambda_{\mathrm{n}, b}$, then this condition is necessary and sufficient. Lötstedt analyzes in [38, Lemma 5.2] a mixed LCP that corresponds to (29) or to (16) written at the multipliers level with the assumption that $\frac{d}{d t}\left(\nabla h_{\mathrm{n}, b}(q)^{T}\right) \dot{q}=0$ and $\frac{d}{d t}\left(\nabla h_{\mathrm{n}, u}(q)^{T}\right) \dot{q}=0^{6}$ (the MLCP in (5.2a)-(5.2c) in [38] is easily obtained from (16) with this assumption). This allows us to relax Assumption 2 to $\operatorname{rank} \nabla h_{\mathrm{n}, b}(q) \leq \min \left(n, m_{b}\right)$ and $\operatorname{rank} \nabla h_{\mathrm{n}, u}(q) \leq \min \left(n, m_{u}\right)$, which guarantee the existence and uniqueness of $\nabla h_{\mathrm{n}, b}(q) \lambda_{\mathrm{n}, b}$ and $\nabla h_{\mathrm{n}, u}(q) \lambda_{\mathrm{n}, u} \cdot{ }^{7}$ We tend to believe that the analysis of (29) using the tools on well-posedness of variational inequalities in [3] could yield similar results in a more general setting.

\section{Systems with sliding Coulomb's friction}

We now turn our attention to systems that are subject to Coulomb's friction. As announced before, we only deal with sliding friction, hence encapsulating the 3D case as well.

\subsection{The Lagrange dynamics with sliding friction}

Using suitable local kinematics frames (see, e.g., [1, §3.3]), we can define the (scalar) tangential velocity $v_{\mathrm{t}, i} \neq 0$ and formulate the sliding Coulomb's friction as

$$
\lambda_{\mathrm{t}, i}=-\mu_{i}\left|\lambda_{\mathrm{n}, i}\right| \xi_{i}, \quad \text { where } \xi_{i} \in\{-1 ; 1\} \text { is unequivocally given by the sign of } v_{\mathrm{t}, i} .
$$

Let $v_{\mathrm{t}, b}$ be the vector that gathers local tangential velocities for the bilaterally constrained contact points, and $v_{\mathrm{t}, u}$ its unilateral counterpart. Then the local operators $H_{\mathrm{t}, b}(q)$ and $H_{\mathrm{t}, u}(q)$ define a local mapping between local and generalized tangential velocities, written as $v_{\mathrm{t}, b}=H_{\mathrm{t}, b}(q)^{T} \dot{q}$ and $v_{\mathrm{t}, u}=H_{\mathrm{t}, u}(q)^{T} \dot{q}$. Let $F_{\mathrm{t}, b}$ denote the generalized force resulting from the contribution of the frictional (tangential) forces acting on the system. From the principle of virtual works it follows that $v_{\mathrm{t}, b}^{T} \lambda_{\mathrm{t}, b}=\dot{q}^{T} H_{\mathrm{t}, b}(q) \lambda_{\mathrm{t}, b}=\dot{q}^{T} F_{\mathrm{t}, b}$. Hence, $F_{\mathrm{t}, b}=H_{\mathrm{t}, b}(q) \lambda_{\mathrm{t}, b}$, and similarly for the unilateral constraints. Then, using (1a) and (1b), we obtain

$$
\begin{aligned}
M(q) \ddot{q}+F(q, \dot{q}, t)= & \nabla h_{\mathrm{n}, b}(q) \lambda_{\mathrm{n}, b}+\nabla h_{\mathrm{n}, u}(q) \lambda_{\mathrm{n}, u}-H_{\mathrm{t}, b}(q)\left[\mu_{b}\right]\left[\xi_{b}\right]\left|\lambda_{\mathrm{n}, b}\right| \\
& -H_{\mathrm{t}, u}(q)\left[\mu_{u}\right]\left[\xi_{u}\right] \lambda_{\mathrm{n}, u},
\end{aligned}
$$

where $\left[x_{b}\right]$ denotes the diagonal matrix $\operatorname{diag}\left(x_{i}\right)$ for $1 \leq i \leq m_{b}$, and $\left[x_{u}\right]$ the diagonal ma$\operatorname{trix} \operatorname{diag}\left(x_{i}\right)$ for $m_{b}+1 \leq i \leq m$, and where $\left|\lambda_{\mathrm{n}, b}\right|=\left(\left|\lambda_{\mathrm{n}, b, 1}\right|\left|\lambda_{\mathrm{n}, b, 2}\right| \ldots\left|\lambda_{\mathrm{n}, b, m_{b}}\right|\right)^{T} \in \mathbb{R}^{m_{b}}$. Note that the unilateral normal forces $\lambda_{\mathrm{n}, u}$ do not need to be expressed in terms of absolute value since they should always remain positive due to the complementarity constraint (1c). Equation (35) serves as a starting point to analyze (1a)-(1d) with sliding Coulomb's friction.

\footnotetext{
${ }^{6}$ Or, more generally, that $\frac{d}{d t}\left(\nabla h_{\mathrm{n}, b}(q)^{T}\right) \dot{q} \in \operatorname{Im}\left(\nabla h_{\mathrm{n}, b}(q)^{T}\right)$ and $\frac{d}{d t}\left(\nabla h_{\mathrm{n}, u}(q)^{T}\right) \dot{q} \in \operatorname{Im}\left(\nabla h_{\mathrm{n}, u}(q)^{T}\right)$.

${ }^{7}$ Theorem 1 in [15] is wrongly used in the proof of [38, Lemma 5.2]; however, this does not call into question the result, which is right.
} 
The Lagrange dynamics with unilateral constraints, Coulomb's friction and impacts as in (35) are very well developed in [36, Chap. 5], whereas their Lyapunov stability is analyzed in detail in [36, Chap. 7]. Since we are dealing with the impactless contact, (35) has to be completed with (1c) and (1d). Notice that $\lambda_{\mathrm{n}, b}^{T} \nabla h_{\mathrm{n}, b}(q)^{T} \dot{q}=0$ since $\nabla h_{\mathrm{n}, b}(q)^{T} \dot{q}=0$. From Proposition 2 we have $0 \leq \lambda_{\mathrm{n}, u, i}(t) \perp \nabla h_{\mathrm{n}, u, i}(q(t))^{T} \dot{q}(t) \geq$ $0 \Leftrightarrow \lambda_{\mathrm{n}, u, i}(t) \in-\partial \Psi_{\mathbb{R}_{+}}\left(\nabla h_{\mathrm{n}, u, i}(q(t))^{T} \dot{q}(t)\right)$ for all $i$ such that $h_{\mathrm{n}, u, i}(q(t))=0$. Suppose that all $m_{u}$ unilateral contacts are active. Then from [51, Theorem 23.9] it follows that $\nabla h_{\mathrm{n}, u}(q) \lambda_{\mathrm{n}, u} \in-\partial \Psi_{T_{\Phi_{u}}(q)}(\dot{q})$ with $T_{\Phi_{u}}(q)=\left\{z \in \mathbb{R}^{n} \mid \nabla h_{\mathrm{n}, u}(q)^{T} z \geq 0\right\}{ }^{8}$ These developments may be used to prove that the contact forces (including tangential ones) dissipate energy, provided that $\lambda_{\mathrm{n}, u}$ and $\lambda_{\mathrm{n}, b}$ exist.

It is worth noting, however, that Coulomb's law cannot be written as an associated law ${ }^{9}$ $[25, \S 4]$. This means that the right-hand side of (35), together with the complementarity conditions, cannot be written compactly as the normal cone to some convex set. De Saxcé's bipotential function [53] allows us to recover an associated form at the local kinematics level, however, at the price of using a modified tangential velocity [1, §3.9.2].

\subsection{Bilaterally constrained systems}

In this section, we analyze different cases and show that various results may be obtained on the well-posedness of the contact problem, depending on the assumptions made.

\subsubsection{All frictional bilateral constraints}

Suppose that $\mathcal{I}_{b}^{0}=\emptyset$, that is, $m_{b}^{\mu}=m_{b}$, and that all contacts are sliding: $\xi_{i} \triangleq \operatorname{sgn}\left(v_{\mathrm{t}, b, i}\right) \in$ $\{-1,1\}$. Using (35), the extension of the KKT system in (4) is

$$
\underbrace{\left(\begin{array}{cc}
M(q) & -\nabla h_{\mathrm{n}, b}(q)+H_{\mathrm{t}, b}(q)\left[\mu_{b} \xi\right]\left[\operatorname{sgn}\left(\lambda_{\mathrm{n}, b}\right)\right] \\
\nabla h_{\mathrm{n}, b}(q)^{T} & 0
\end{array}\right)}_{\triangleq_{M_{b \mu \xi}(q)}}\left(\begin{array}{c}
\ddot{q} \\
\lambda_{\mathrm{n}, b}
\end{array}\right)=\left(\begin{array}{c}
-F(q, \dot{q}, t) \\
-\frac{d}{d t}\left(\nabla h_{\mathrm{n}, b}(q)^{T}\right) \dot{q}
\end{array}\right),
$$

and we may denote the matrix in the left-hand side of (36) as $M_{b \mu \xi}(q)$. Comparing with (4), we see that friction modifies the system matrix, not its right-hand side. Bilateral friction introduces a nonlinearity or rather a piecewise linearity in the variable $\lambda_{\mathrm{n}, b}$.

A direct way of analyzing the existence and uniqueness of solutions $\left(\ddot{q}, \lambda_{n, b}\right)$ of (36) is to form the analog of (7),

$$
A_{n b}(q) \lambda_{n, b}-A_{t b}(q)[\mu \xi]\left|\lambda_{n, b}\right|+w_{b}(q, \dot{q}, t)=0,
$$

where the absolute value is meant componentwise, and $A_{t b}(q) \triangleq \nabla h_{\mathrm{n}, b}(q)^{T} M(q)^{-1} H_{\mathrm{t}, \mathrm{b}}(q)$ is a Delassus-like matrix coupling normal and tangential frictional effects. By inspecting each of the $2^{m_{b}}$ possible signs of $\lambda_{n, b}$ we obtain $2^{m_{b}}$ linear systems. We may solve each such system and obtain a candidate solution $\lambda_{n, b}$. If the signs of the newly computed $\lambda_{n, b}$ coincide with those of the assumption, then a solution has indeed been found; otherwise, it

\footnotetext{
${ }^{8}$ The notation for this set is chosen to recall that under some constraint qualification (like MangasarianFromovitz), $T_{\Phi_{u}}(q)$ is the tangent cone polar to the normal cone.

${ }^{9} \mathrm{~A}$ contact law is associated if it can be expressed as an inclusion in the subdifferential of a convex, proper function, i.e., it admits a convex pseudo-potential.
} 
is rejected. If no solution or several solutions are found, then the system is said to be wedged or jammed. Such an exhaustive procedure would yield necessary and sufficient conditions on the parameter values for a unique solution of problem (37) to exist (see [4] in the case of a single contact). However, in practice, it becomes intractable for systems with more than a few contact points.

In the rest of this section, we present two alternative ways to derive sufficient conditions for Eq. (36) to have a unique solution. Problem (37) can be treated as a perturbation of the frictionless problem (7) and the nonlinearity introduced by bilateral friction can be treated via a fixed point argument or via complementarity theory.

Proposition 9 Let $(q, \dot{q})$ and $F(q, \dot{q}, t)$ be given. Let $A_{n b}(q)$ be positive definite. Suppose that all contacts are sliding $\left(v_{\mathrm{t}, b, i} \neq 0\right)$ and that

$$
\max _{1 \leq i \leq m} \mu_{i}<\mu_{\max }^{b}(q)=\frac{\sigma_{\min }\left(A_{n b}(q)\right)}{\sigma_{\max }\left(A_{t b}(q)\right)}
$$

Then the bilateral sliding friction problem (36) has a unique solution $\left(\ddot{q}, \lambda_{\mathrm{n}, b}\right)$.

Proof Under the rank assumption made in the proposition, system (36) is equivalent to

$$
\left\{\begin{array}{l}
\ddot{q}=M(q)^{-1} \nabla h_{\mathrm{n}, b}(q) \lambda_{n, b}-M(q)^{-1} H_{\mathrm{t}, b}(q)\left[\mu_{b}\right]\left[\operatorname{sgn}\left(v_{\mathrm{t}, b}\right)\right]\left|\lambda_{\mathrm{n}, b}\right|-M(q)^{-1} F(q, \dot{q}, t), \\
A_{n b}(q) \lambda_{n, b}-A_{t b}(q)[\mu \xi]\left|\lambda_{n, b}\right|+w_{b}(q, \dot{q}, t)=0 .
\end{array}\right.
$$

Since $A_{n b}(q)$ can be inverted, $\lambda_{\mathrm{n}, b}$ is a solution of (36) if and only if $\lambda_{\mathrm{n}, b}=A_{n b}^{-1} A_{t b}[\mu \xi] \times$ $\left|\lambda_{\mathrm{n}, b}\right|-A_{n b}^{-1} w_{b}$. Letting $T(x) \triangleq A_{n b}^{-1} A_{t b}[\mu \xi]|x|-A_{n b}^{-1} w_{b}$, we will show that the mapping $T(\cdot)$ is contracting under (38). Indeed, we have:

$$
\begin{aligned}
\|T(x)-T(y)\|_{2} & =\left\|A_{n b}^{-1} A_{t b}[\mu \xi](|x|-|y|)\right\|_{2} \leq\left\|A_{n b}^{-1} A_{t b}[\mu \xi]\right\|_{2}\||x|-|y|\|_{2} \\
& \leq\left\|A_{n b}^{-1} A_{t b}[\mu \xi]\right\|_{2}\|x-y\|_{2}
\end{aligned}
$$

for any $x, y \in \mathbb{R}^{m_{b}}$. By the property of induced matrix norms [6, Proposition 9.4.3] and the fact that $\|[a]\|_{2}=\max _{i}\left|a_{i}\right|$ we have $\max _{1 \leq i \leq m} \mu_{i}<\frac{1}{\left\|A_{n b}^{-1}\right\|_{2}\left\|A_{t b}\right\|_{2}}$, which implies $\left\|A_{n b}^{-1} A_{t b}[\mu \xi]\right\|_{2}<1$. Hence, $T(\cdot)$ is contracting. Using the Banach fixed point theorem, we conclude that a unique solution $\lambda_{\mathrm{n}, b}$ to (39) exists. The existence and uniqueness of the acceleration in (39) follows from that of $\lambda_{\mathrm{n}, b}$.

The upper bound for the friction coefficients in Proposition 9 is necessary and sufficient in the case of a single contact. In the general case (several contact points), it is only sufficient. Depending on the mechanical system under consideration, the friction bound may or may not be conservative. Note, however, that the actual critical friction bound over which Painlevé-like paradoxes occur may, in fact, be arbitrarily small [35].

The following result is similar to Proposition 9. It has a stronger requirements on the friction bounds, but the proof uses complementarity theory rather than a fixed point argument, and the decomposition introduced is a key in decoupling frictional and frictionless parts in the mixed unilateral/bilateral case studied in Sect. 3.2.2. 
Proposition 10 Let $(q, \dot{q})$ and $F(q, \dot{q}, t)$ be given. Let $A_{n b}(q)$ be positive definite. Suppose that all contacts are sliding $\left(v_{\mathrm{t}, b, i} \neq 0\right)$ and that

$$
\max _{1 \leq i \leq m} \mu_{i}<\frac{1}{3} \mu_{\max }^{b}(q)
$$

where $\mu_{\max }^{b}(q)$ is defined in (38). Then the bilateral sliding friction problem (36) has a unique solution $\left(\ddot{q}, \lambda_{\mathrm{n}, b}\right)$.

Proof The proof consists of three steps: reformulating (37) as a horizontal LCP $\left(\mathrm{hLCP}^{10}\right)$, casting the hLCP matrix as a perturbation of a positive definite matrix, and applying Corollary 3 in Appendix D. We can write $\left[\operatorname{sgn}\left(\lambda_{\mathrm{n}, b}\right)\right] \lambda_{\mathrm{n}, b}=\left|\lambda_{\mathrm{n}, b}\right|$. Following [27], let us introduce $\lambda^{+} \triangleq \frac{\left|\lambda_{\mathrm{n}, b}\right|+\lambda_{\mathrm{n}, b}}{2}$ and $\lambda^{-} \triangleq \frac{\left|\lambda_{\mathrm{n}, b}\right|-\lambda_{\mathrm{n}, b}}{2}$. Solving (37) boils down to find $\lambda^{+}$and $\lambda^{-}$such that

$$
\left\{\begin{array}{l}
\left(A_{n b}(q)-A_{t b}(q)\left[\mu_{b} \xi\right]\right) \lambda^{+}-\left(A_{n b}(q)+A_{t b}(q)\left[\mu_{b} \xi\right]\right) \lambda^{-}+w_{b}(q, \dot{q}, t)=0, \\
0 \leq \lambda^{+} \perp \lambda^{-} \geq 0 .
\end{array}\right.
$$

As pointed out in [27], a solution to the hLCP (41) exists and is unique if and only if $A_{n b}-$ $A_{t b}\left[\mu_{b} \xi\right]$ is invertible and $\left(A_{n b}-A_{t b}\left[\mu_{b} \xi\right]\right)^{-1}\left(A_{n b}+A_{t b}\left[\mu_{b} \xi\right]\right)$ is a P-matrix. The upper bound on the friction coefficient assures $\left\|A_{n b}^{-1} A_{t b}\left[\mu_{b} \xi\right]\right\|_{2} \leq\left\|A_{n b}^{-1} A_{t b}\right\|_{2}\left\|\left[\mu_{b}\right]\right\|_{2} \leq \frac{1}{3}<1$, securing that $I-A_{n b}^{-1} A_{t b}\left[\mu_{b} \xi\right]$ is nonsingular and that its inverse can be written using Taylor series expansion. The hLCP matrix, whose positive definiteness we want to enforce, can be rewritten as $\left(A_{n b}-A_{t b}\left[\mu_{b} \xi\right]\right)^{-1}\left(A_{n b}+A_{t b}\left[\mu_{b} \xi\right]\right)=\left(I-A_{n b}^{-1} A_{t b}\left[\mu_{b} \xi\right]\right)^{-1} A_{n b}^{-1} A_{n b}(I+$ $\left.A_{n b}^{-1} A_{t b}\left[\mu_{b} \xi\right]\right)=\left(\sum_{k=0}^{+\infty}\left(A_{n b}^{-1} A_{t b}\left[\mu_{b} \xi\right]\right)^{k}\right)\left(I+A_{n b}^{-1} A_{t b}\left[\mu_{b} \xi\right]\right)=I+\underbrace{2 \sum_{k=1}^{+\infty}\left(A_{n b}^{-1} A_{t b}\left[\mu_{b} \xi\right]\right)^{k}}_{\triangleq_{K_{\mu}}(q, \dot{q})}$.

From Corollary 3 in Appendix D it follows that if $\left\|K_{\mu}(q, \dot{q})\right\|_{2}<1$, then $I+K_{\mu}(q, \dot{q}) \succ 0 .{ }^{11}$ The upper bound $\frac{1}{3} \mu_{\max }^{b}(q)$ is chosen specifically so that the following condition holds: $\left\|K_{\mu}(q, \dot{q})\right\| \leq 2 \sum_{k=1}^{+\infty}\left\|A_{n b}^{-1} A_{t b}\right\|^{k}\left\|\left[\mu_{b}\right]\right\|^{k}<2 \sum_{k=1}^{+\infty}\left\|A_{n b}^{-1} A_{t b}\right\|^{k} \frac{1}{3^{k}\left\|A_{n b}^{-1} A_{t b}\right\|^{k}}<1$.

Example (A bilaterally constrained system with two frictional contacts) We now consider the so-called Painlevé-Klein system depicted in Fig. 1(a), where a rod is subjected to two bilateral frictional constraints with friction coefficients $\mu_{1}, \mu_{2}$. Proposition 9 can be used to study this system. It has been long known [47] that the contact LCP of this system has a unique solution for the multipliers if and only if $\left|\mu_{1}-\mu_{2}\right|<\frac{2}{\tan \theta}$; this necessary and sufficient condition can be thought of as defining a region in the $\left(\mu_{1}, \mu_{2}\right)$ parameter plane, and it is depicted in dashes in Fig. 1(b). In contrast, the merely sufficient condition of Proposition 9 defines a smaller region in the friction parameter plane (the dotted square in Fig. 1(b)). The proposition states that if $\max \left(\mu_{1}, \mu_{2}\right)<\frac{\sigma_{\min }\left(A_{n b}(\theta)\right)}{\sigma_{\max }\left(A_{t b}(\theta)\right)}$, then the multiplier vector exists and is unique. Explicitly computing the singular values for a rod of length $1 \mathrm{~m}$ and mass $1 \mathrm{~kg}$ yields

$$
\begin{aligned}
\max \left(\mu_{1}, \mu_{2}\right) & <\mu_{\max }^{b}(\theta) \\
& \triangleq \frac{2}{3 \sqrt{-\cos (4 \theta)+1}} \sqrt{-\left(3 \cos ^{2}(\theta)+1\right)\left|3 \cos ^{2}(\theta)-1\right|+9 \cos ^{4}(\theta)+1,}
\end{aligned}
$$

\footnotetext{
${ }^{10}$ See [1, Definition 12.23].

${ }^{11}$ A positive definite matrix is also a $\mathrm{P}$-matrix.
} 


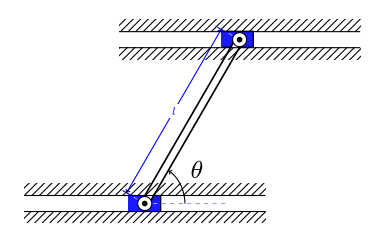

(a) Rod with two bilateral constraints

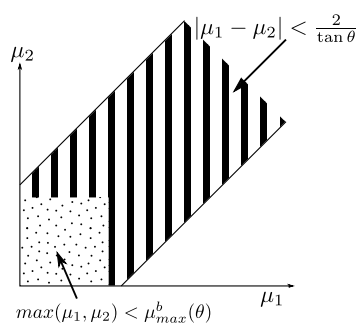

(b) Conservativeness of $\mu_{b}^{\max }(\theta)$

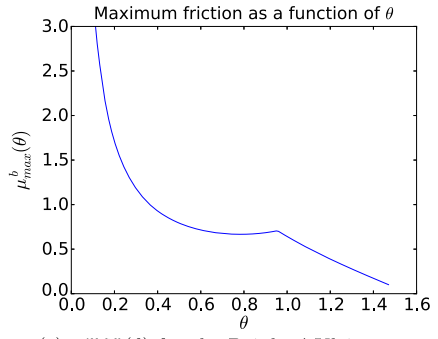

(c) $\mu_{b}^{\max }(\theta)$ for the Painlevé-Klein system

Fig. 1 Friction bound for the Painlevé-Klein system

and it can be checked numerically that $\mu_{\max }^{b}(\theta)<\frac{2}{\tan \theta}$ for all $\left.\theta \in\right] 0 ; \pi / 2[$. In Fig. 1(c), $\mu_{b}^{\max }(\theta)$ is plotted as a function of $\theta$. If the system is at a certain state $\theta$ and the friction at each guide is less than the value $\mu_{b}^{\max }(\theta)$ on the curve, then the contact force exists and is unique.

\subsubsection{Mixed frictional/frictionless contacts}

Let us now assume that $\mathcal{I}_{b}^{0} \neq \emptyset$ and $\mathcal{I}_{b}^{\mu} \neq \emptyset$ : some of the bilateral contacts are frictionless, the others are frictional. We rewrite (35) in its index 1 form:

$$
\left\{\begin{array}{l}
M(q) \ddot{q}+F(q, \dot{q}, t)=\nabla h_{\mathrm{n}, b}^{0}(q) \lambda_{\mathrm{n}, b}^{0}+\nabla h_{\mathrm{n}, b}^{\mu}(q) \lambda_{\mathrm{n}, b}^{\mu}+H_{\mathrm{t}, b}^{\mu}(q)\left[\mu_{b}\right][\xi]\left[\operatorname{sgn}\left(\lambda_{\mathrm{n}, b}^{\mu}\right)\right] \lambda_{\mathrm{n}, b}^{\mu}, \\
\nabla h_{\mathrm{n}, b}^{0}(q)^{T} \ddot{q}+\frac{d}{d t}\left(\nabla h_{\mathrm{n}, b}^{0}(q)^{T}\right) \dot{q}=0, \quad \nabla h_{\mathrm{n}, b}^{\mu}(q)^{T} \ddot{q}+\frac{d}{d t}\left(\nabla h_{\mathrm{n}, b}^{\mu}(q)^{T}\right) \dot{q}=0
\end{array}\right.
$$

In a matrix form, we get an extension of (36):

$$
\begin{aligned}
& \left(\begin{array}{ccc}
M(q) & -\nabla h_{\mathrm{n}, b}^{0}(q) & -\nabla h_{\mathrm{n}, b}^{\mu}(q)+H_{\mathrm{t}, b}^{\mu}(q)\left[\mu_{b}\right][\xi]\left[\operatorname{sgn}\left(\lambda_{\mathrm{n}, b}^{\mu}\right)\right] \\
\nabla h_{\mathrm{n}, b}^{0}(q)^{T} & 0 & 0 \\
\nabla h_{\mathrm{n}, b}^{\mu}(q)^{T} & 0 & 0
\end{array}\right)\left(\begin{array}{c}
\ddot{q} \\
\lambda_{\mathrm{n}, b}^{0} \\
\lambda_{\mathrm{n}, b}^{\mu}
\end{array}\right) \\
& =\left(\begin{array}{c}
-F(q, \dot{q}, t) \\
-\frac{d}{d t}\left(\nabla h_{\mathrm{n}, b}^{0}(q)^{T}\right) \dot{q} \\
-\frac{d}{d t}\left(\nabla h_{\mathrm{n}, b}^{\mu}(q)^{T}\right) \dot{q}
\end{array}\right) .
\end{aligned}
$$

Similarly to all frictional cases, assuming that $M(q) \succ 0$ and that the nonfrictional constraints are independent, we obtain a reduced system, where the only unknown is $\lambda_{\mathrm{n}, b}^{\mu}$. Under these assumptions, system (42) is equivalent to (the argument $q$ is dropped)

$$
\left\{\begin{aligned}
& \ddot{q}=- M^{-1} F(q, \dot{q}, t)+M^{-1} \nabla h_{\mathrm{n}, b}^{0} \lambda_{\mathrm{n}, b}^{0}+M^{-1} \nabla h_{\mathrm{n}, b}^{\mu} \lambda_{\mathrm{n}, b}^{\mu}-M^{-1} H_{\mathrm{t}, b}^{\mu}\left[\mu_{b} \xi\right]\left|\lambda_{\mathrm{n}, b}^{\mu}\right| \\
& \lambda_{\mathrm{n}, b}^{0}=-\left(A_{n b}^{00}\right)^{-1} A_{n b}^{0 \mu} \lambda_{n b}^{\mu}+\left(A_{n b}^{00}\right)^{-1} A_{t b}^{0 \mu}\left[\mu_{b} \xi\right]\left|\lambda_{\mathrm{n}, b}^{\mu}\right| \\
& \quad+\left(A_{n b}^{00}\right)^{-1}\left(\left(\nabla h_{\mathrm{n}, b}^{0}\right)^{T} M^{-1} F-\frac{d}{d t}\left(\left(\nabla h_{\mathrm{n}, b}^{0}\right)^{T}\right) \dot{q}\right) \\
&\left(A_{n b}^{\mu \mu}-\left(A_{n b}^{0 \mu}\right)^{T}\left(A_{n b}^{00}\right)^{-1} A_{n b}^{0 \mu}\right) \lambda_{\mathrm{n}, b}^{\mu}-\left(A_{t b}^{\mu \mu}-\left(A_{n b}^{0 \mu}\right)^{T}\left(A_{n b}^{00}\right)^{-1} A_{t b}^{0 \mu}\right)[\mu \xi]\left|\lambda_{\mathrm{n}, b}^{\mu}\right|+\tilde{w}_{b}=0,
\end{aligned}\right.
$$

where $A_{n b}^{00}(q)=\nabla h_{\mathrm{n}, b}^{0}(q)^{T} M(q)^{-1} \nabla h_{\mathrm{n}, b}^{0}(q)$ is the matrix of normal/normal frictionless couplings, $A_{n b}^{0 \mu}(q)=\nabla h_{\mathrm{n}, b}^{0}(q)^{T} M(q)^{-1} \nabla h_{\mathrm{n}, b}^{\mu}(q)$ is the matrix of normal friction- 
less/normal frictional couplings, $A_{t b}^{0 \mu}(q)=\nabla h_{\mathrm{n}, b}^{0}(q)^{T} M(q)^{-1} H_{\mathrm{t}, b}^{\mu}(q)$ is the matrix of normal frictionless/tangential frictional couplings, $A_{t b}^{\mu \mu}(q)=\nabla h_{\mathrm{n}, b}^{\mu}(q)^{T} M(q)^{-1} H_{\mathrm{t}, b}^{\mu}(q)$ is the matrix of normal frictional/tangential frictional couplings, and, finally, $\tilde{w}_{b}(q, \dot{q}, t) \triangleq$ $A_{n b}^{0 \mu}\left(A_{n b}^{00}\right)^{-1}\left(\left(\nabla h_{\mathrm{n}, b}^{0}\right)^{T} M^{-1} F-\frac{d}{d t}\left(\left(\nabla h_{\mathrm{n}, b}^{0}\right)^{T}\right) \dot{q}\right)-\left(\left(\nabla h_{\mathrm{n}, b}^{\mu}\right)^{T} M^{-1} F-\frac{d}{d t}\left(\left(\nabla h_{\mathrm{n}, b}^{\mu}\right)^{T}\right) \dot{q}\right)$ is the vector collecting all remaining terms in the last equation. Hence, the problem is reduced to studying essentially the same equation as (37) in the all frictional case (which was itself an extension of (7)):

$$
\tilde{A}_{n b}(q) \lambda_{\mathrm{n}, b}^{\mu}-\tilde{A}_{t b}(q)[\mu \xi]\left|\lambda_{\mathrm{n}, b}^{\mu}\right|+\tilde{w}_{b}(q, \dot{q}, t)=0,
$$

where $\tilde{A}_{n b} \triangleq A_{n b}^{\mu \mu}-\left(A_{n b}^{0 \mu}\right)^{T}\left(A_{n b}^{00}\right)^{-1} A_{n b}^{0 \mu}$ and $\tilde{A}_{t b} \triangleq A_{t b}^{\mu \mu}-\left(A_{n b}^{0 \mu}\right)^{T}\left(A_{n b}^{00}\right)^{-1} A_{t b}^{0 \mu}$. It is important to observe that $\tilde{A}_{n b}(q)$ is nothing but the Schur complement of $A_{n b}^{\mu \mu}(q)$ in the matrix $\left(\nabla h_{\mathrm{n}, b}^{0} \nabla h_{\mathrm{n}, b}^{\mu}\right)^{T} M^{-1}\left(\nabla h_{\mathrm{n}, b}^{0} \nabla h_{\mathrm{n}, b}^{\mu}\right)$. So $\tilde{A}_{n b}(q) \succ 0$ and $A_{n b}^{00}(q) \succ 0$ if and only if $\left(\nabla h_{\mathrm{n}, b}^{0} \nabla h_{\mathrm{n}, b}^{\mu}\right)^{T} M^{-1}\left(\nabla h_{\mathrm{n}, b}^{0} \nabla h_{\mathrm{n}, b}^{\mu}\right) \succ 0$. Hence, if $\left(\nabla h_{\mathrm{n}, b}^{0}(q) \nabla h_{\mathrm{n}, b}^{\mu}(q)\right)$ is of full rank, then $\tilde{A}_{n b}(q)$ is invertible. We deduce the following result.

Proposition 11 Let $(q, \dot{q})$ and $F(q, \dot{q}, t)$ be given, and $v_{\mathrm{t}, b, i} \neq 0$ for all $i \in \mathcal{I}_{b}^{\mu}$. Assume that the matrix

$$
\left(\nabla h_{\mathrm{n}, b}^{0}(q) \nabla h_{\mathrm{n}, b}^{\mu}(q)\right)^{T} M(q)^{-1}\left(\nabla h_{\mathrm{n}, b}^{0}(q) \nabla h_{\mathrm{n}, b}^{\mu}(q)\right)
$$

is positive definite. If, furthermore, $\max _{1 \leq i \leq m} \mu_{i}<\frac{\sigma_{\min }\left(\tilde{A}_{n b}(q)\right)}{\sigma_{\max }\left(\tilde{A}_{t b}(q)\right)}$, then the solution $\left(\ddot{q}, \lambda_{\mathrm{n}, b}^{0}, \lambda_{\mathrm{n}, b}^{\mu}\right)$ of system (42) exists and is unique.

The proof is almost the same as that of Proposition 9: the existence and uniqueness are obtained by a fixed point argument, and the bound on the matrix norm guarantees that an affine operator is contracting. Assumption (46) is there to ensure that $\tilde{A}_{n b}(q)$ is invertible.

\subsection{Unilaterally constrained systems}

We now assume that $m_{b}=0$ and $m_{u} \geq 1$. The unilateral sliding friction problem then consists in finding the accelerations and contact forces $\left(\ddot{q}, \lambda_{\mathrm{n}, u}\right)$ that are the solutions of the MLCP

$$
\left\{\begin{array}{l}
M(q) \ddot{q}+F(q, \dot{q}, t)=\left(\nabla h_{\mathrm{n}, u}(q)-H_{\mathrm{t}, u}(q)[\mu][\xi]\right) \lambda_{\mathrm{n}, u}, \\
0 \leq \nabla h_{\mathrm{n}, u}(q)^{T} \ddot{q}+\frac{d}{d t}\left(\nabla h_{\mathrm{n}, u}(q)^{T}\right) \dot{q} \perp \lambda_{\mathrm{n}, u} \geq 0 .
\end{array}\right.
$$

\subsubsection{Augmented contact LCP}

If $M(q) \succ 0$, then the problem boils down to an LCP. Let us introduce the matrix

$$
\begin{aligned}
A_{u, \mu, \xi}(q) & \triangleq \nabla h_{\mathrm{n}, u}(q)^{T} M(q)^{-1}\left(\nabla h_{\mathrm{n}, u}(q)-H_{\mathrm{t}, u}(q)\left[\mu_{u}\right][\xi]\right) \\
& =A_{n u}(q)-A_{t u}(q)\left[\mu_{u}\right][\xi]
\end{aligned}
$$

with $\xi_{i}=\operatorname{sgn}\left(v_{\mathrm{t}, u, i}\right), v_{t, u, i} \neq 0$ for $1 \leq i \leq m_{u}$. Then the frictional contact LCP, which extends (9), is given by

$$
0 \leq \lambda_{\mathrm{n}, u} \perp A_{u, \mu, \xi}(q) \lambda_{\mathrm{n}, u}+w_{u}(q, \dot{q}, t) \geq 0
$$

with $w_{u}(q, \dot{q}, t)$ in (10). 
Proposition 12 Let $(q, \dot{q})$ and $F(q, \dot{q}, t)$ be given, and $v_{t, u, i} \neq 0$ for $1 \leq i \leq m_{u}$. Let $A_{n u}(q)$ be positive definite and suppose that

$$
\max _{1 \leq i \leq m} \mu_{i}<\mu_{\max }^{u}(q) \triangleq \frac{\sigma_{\min }\left(A_{n u}(q)\right)}{\sigma_{\max }\left(A_{t u}(q)\right)} .
$$

Then the matrix $A_{u, \mu, \xi}(q)$ is positive definite, and the contact LCP (50) has always a unique solution $\lambda_{\mathrm{n}, u}(q, \dot{q}, t, \mu)$, which is a Lipschitz continuous function of $w_{u}(q, \dot{q}, t)$.

The last result follows from basic complementarity theory [16]. The fact that (51) guarantees that $A_{u, \mu, \xi}(q) \succ 0$ may be proved using Corollary 3 in Appendix D. Proposition 12 thus states that whatever the status of the tangential contacts may be (provided that they are sliding), $\lambda_{\mathrm{n}, u}$ can always be calculated uniquely. As alluded to above, extending Propositions 3 and 7 requires some care because even if the LCP matrix may still be positive (semi) definite when a sliding friction is involved (because the "disturbance" due to friction is small), its symmetry is usually lost. Symmetry is an important property (see Theorem 1(d) in Appendix B). When $A_{u, \mu, \xi}(q) \succeq 0$ but not symmetric, then using [16, Theorem 3.1.7], various properties of the solutions may be derived (however, Proposition 3(ii) cannot be stated due to the lack of symmetry): if $\lambda_{\mathrm{n}, u}^{1}$ and $\lambda_{\mathrm{n}, u}^{2}$ are two solutions of the LCP (50), then $\lambda_{\mathrm{n}, u}^{1, T}\left(A_{u, \mu, \xi}(q)-A_{u, \mu, \xi}(q)^{T}\right) \lambda_{\mathrm{n}, u}^{2}=\left(\lambda_{\mathrm{n}, u}^{2}-\lambda_{\mathrm{n}, u}^{1}\right) w_{u}(q, \dot{q}, t)$. Also, solvability implies that the set of solutions is polyhedral. Using a characterization of copositive matrices [8, §4.41], we may state the following, which may be seen as an extension of Proposition 3(iii, iv).

Corollary 2 Suppose that there exists $[\xi]$ such that the matrix $A_{t u}(q)\left[\mu_{u}\right][\xi]$ is elementwise nonpositive. Then, provided that $\left(0 \leq \lambda_{\mathrm{n}, u} \perp A_{u, \mu, \xi}(q) \lambda_{\mathrm{n}, u} \geq 0 \Rightarrow \lambda_{\mathrm{n}, u}^{T} w_{u}(q, \dot{q}, t) \geq 0\right)$, the LCP in (50) is solvable.

Indeed, from $[8, \S 4.41]$, the sum of a symmetric positive semidefinite together with an elementwise nonnegative matrices is copositive. Hence, under the stated conditions, $A_{u, \mu, \xi}(q)$ is copositive. Then apply Theorem 2 in Appendix C. Thus, Corollary 2 boils down to testing whether tangential velocities signs exist such that the perturbation matrix has all its entries nonnegative. As simple examples like the classical Painlevé sliding rod show [21], this may be the case. Obviously, reversing the tangential velocities signs may destroy the copositivity of $A_{u, \mu, \xi}(q)$ and may induce singularities like Painlevé paradoxes. It is worth noting that a direct extension of item (v) in Proposition 3 is generically not possible because $A_{u, \mu, \xi}(q)$ is nonsymmetric. Thus, the characterization of the solutions of the homogeneous LCP is not straightforward.

Notice that in a sticking regime where $v_{\mathrm{t}}=0$, the LCP in (50) is not sufficient to calculate $\lambda_{\mathrm{n}, u}$ uniquely because the selections of the set-valued term $\operatorname{sgn}\left(v_{\mathrm{t}}\right)=\operatorname{sgn}(0)=[-1,1]$ are still to be determined. We have to resort to Coulomb's law at the acceleration level [23, 48] to determine which type of transition the system undergoes.

\subsubsection{Inclusions and Gauss' principle}

It is of interest to investigate whether or not some minimization problem for the acceleration may be obtained in the sliding frictional case. The starting point is the MLCP in (47), which is transformed using (49) into an inclusion that generalizes (14). The question is then whether or not an extension of (15) exists or not. Let $\ddot{\eta} \triangleq \nabla h_{\mathrm{n}, u}(q)^{T} \ddot{q}+\frac{d}{d t}\left(\nabla h_{\mathrm{n}, u}(q)^{T}\right) \dot{q}$, 
and $M(q) \succ 0$. Using similar tools from convex analysis as in the foregoing sections (see (65)), we obtain:

$$
\underbrace{\ddot{\eta}-\frac{d}{d t}\left(\nabla h_{\mathrm{n}, u}(q)^{T}\right) \dot{q}+\nabla h_{\mathrm{n}, u}(q)^{T} M(q)^{-1} F(q, \dot{q}, t)}_{\triangleq \bar{F}_{u, \mu, \xi}(q, \dot{q}, t)} \in-A_{u, \mu, \xi}(q) \partial \Psi_{\mathbb{R}_{+}^{m_{u}}}(\ddot{\eta}) .
$$

Let the conditions of Proposition 12 be satisfied. Then we have

$$
A_{u, \mu, \xi}(q) \partial \Psi_{\mathbb{R}_{+}^{m_{u}}}(\ddot{\eta})=A_{u, \mu, \xi}(q) \partial \Psi_{\mathbb{R}_{+}^{m_{u}}}\left(A_{u, \mu, \xi}(q)^{T} A_{u, \mu, \xi}(q)^{-T} \ddot{\eta}\right)=N_{\mathcal{E}(q)}(\ddot{\zeta})
$$

with $\ddot{\zeta}=A_{u, \mu, \xi}(q)^{-T} \ddot{\eta}$, where $\mathcal{E}(q)=\left\{z \in \mathbb{R}^{m_{u}} \mid A_{u, \mu, \xi}(q)^{T} z \geq 0\right\}$ is a nonempty closed convex (polyhedral) set, and we used the chain rule of convex analysis [51, Theorem 23.9]. Inclusion (52) is then equivalently rewritten as

$$
A_{u, \mu, \xi}(q)^{T} \ddot{\zeta}+F_{u, \mu, \xi}(q, \dot{q}, t) \in-N_{\mathcal{E}(q)}(\ddot{\zeta}),
$$

where $F_{u, \mu, \xi}(q, \dot{q}, t)=A_{u, \mu, \xi}(q)^{T} \bar{F}_{u, \mu, \xi}(q, \dot{q}, t)$. The crucial fact here is that $A_{u, \mu, \xi}(q) \succ 0$ is not symmetric. However, from $[19, \S 1.1]$ it follows that inclusion (53) is equivalent to the variational inequality

$$
\text { Find } \ddot{\zeta} \in \mathcal{E}(q):\left\langle A_{u, \mu, \xi}(q)^{T} \ddot{\zeta}+F_{u, \mu, \xi}(q, \dot{q}, t), z-\ddot{\zeta}\right\rangle \geq 0 \quad \text { for all } z \in \mathcal{E}(q) \text {. }
$$

Since $A_{u, \mu, \xi}(q) \succ 0$, it follows that the mapping $x \rightarrow A_{u, \mu, \xi}(q)^{T} x+F_{u, \mu, \xi}(q, \dot{q}, t)$ is strongly monotone [19, p. 155], so by [19, Theorem 2.3.3] the variational inequality in (54) has a unique solution $\ddot{\zeta}^{\star}$. This solution also satisfies [19, p. 158]

$$
\ddot{\zeta}^{\star}=\operatorname{proj}\left[\mathcal{E}(q) ; A_{u, \mu, \xi}(q)^{T} \ddot{\zeta}^{\star}+F_{u, \mu, \xi}(q, \dot{q}, t)\right] .
$$

Equations (53) and (55) may be considered as the counterpart of (14) and (15), or (21) and (26), or (27) and (28) for unilateral constraints with sliding Coulomb's friction. Consider the LCP in (50): the extension of (11), which is the minimization associated with the LCP (9), is not clear due again to the lack of symmetry of $A_{u, \mu, \xi}(q)$.

\subsection{Unilaterally/bilaterally constrained systems}

Let us consider again systems as in Sect. 2.3, but with friction at the contacts.

\subsubsection{All contacts with friction}

Let us first assume that all contacts are frictional, that is, $\mathcal{I}_{b}^{0}=\mathcal{I}_{u}^{0}=\emptyset$. Let us consider (1a)-(1d) in its index 1 form, that is, with the acceleration constraints $\nabla h_{\mathrm{n}, b}(q)^{T} \ddot{q}+$ $\frac{d}{d t}\left(\nabla h_{\mathrm{n}, b}(q)^{T}\right) \dot{q}=0$ and $0 \leq h_{\mathrm{n}, u}(q) \perp \nabla h_{\mathrm{n}, u}(q)^{T} \ddot{q}+\frac{d}{d t}\left(\nabla h_{\mathrm{n}, u}(q)^{T}\right) \dot{q} \geq 0$. The dynamics in (1a)-(1d) becomes

$$
\left\{\begin{aligned}
& M(q) \ddot{q}= \nabla h_{\mathrm{n} b}(q) \lambda_{\mathrm{n}, b}-H_{\mathrm{t}, b}(q)\left[\mu_{b} \xi_{b}\right]\left|\lambda_{\mathrm{n}, b}\right|+\nabla h_{\mathrm{n} u}(q) \lambda_{\mathrm{n}, u}-H_{\mathrm{t}, u}(q)\left[\mu_{u} \xi_{u}\right] \lambda_{\mathrm{n}, u} \\
&-F(q, \dot{q}, t), \\
& \nabla h_{\mathrm{n}, b}(q)^{T} \ddot{q}+\frac{d}{d t}\left(\nabla h_{\mathrm{n}, b}(q)^{T}\right) \dot{q}=0, \\
& 0 \leq \lambda_{\mathrm{n}, u} \perp \nabla h_{\mathrm{n} u}(q)^{T} \ddot{q}+\frac{d}{d t}\left(\nabla h_{\mathrm{n}, u}(q)^{T}\right) \dot{q} \geq 0 .
\end{aligned}\right.
$$


By inverting the mass matrix, $\ddot{q}$ can be expressed as a function of the multipliers. Introducing $\lambda^{+}=\frac{\left|\lambda_{n b}\right|+\lambda_{n b}}{2}$ and $\lambda^{-}=\frac{\left|\lambda_{n, b}\right|-\lambda_{n, b}}{2}$ as in Proposition 10 casts the piecewise linearity induced by the absolute value into a complementarity formalism. The problem is thus transformed to determining whether for arbitrary parameter values and arbitrary vectors $w_{1}, w_{2}$, the following MLCP has a unique solution $\left(\lambda^{+}(q, \dot{q}), \lambda^{-}(q, \dot{q}), \lambda_{\mathrm{n}, u}(q, \dot{q})\right):^{12}$

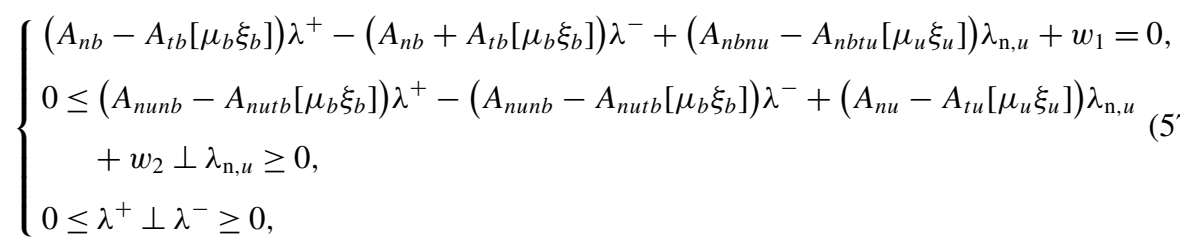

where

$$
w_{1}(q, \dot{q}, t) \triangleq-\nabla h_{\mathrm{n}, b}(q)^{T} M(q)^{-1} F(q, \dot{q}, t)+\frac{d}{d t}\left(\nabla h_{\mathrm{n}, b}(q)^{T}\right) \dot{q}
$$

and

$$
w_{2}(q, \dot{q}, t) \triangleq-\nabla h_{\mathrm{n}, u}(q)^{T} M(q)^{-1} F(q, \dot{q}, t)+\frac{d}{d t}\left(\nabla h_{\mathrm{n}, u}(q)^{T}\right) \dot{q}
$$

possess the same structure as $w_{u}(q, \dot{q}, t)$ in (10). Assume that the conditions of Proposition 10 hold. Then $A_{n b}(q)-A_{t b}(q)\left[\mu_{b} \xi_{b}\right] \succ 0$, and the problem is to determine the existence and uniqueness of a solution $\left(\lambda^{-}(q, \dot{q}), \lambda_{\mathrm{n}, u}(q, \dot{q})\right)$ (for arbitrary $\left.q, \dot{q}\right)$ of the following LCP (the argument $q$ is dropped):

$$
0 \leq\left(\begin{array}{c}
\lambda^{-} \\
\lambda_{\mathrm{n}, u}
\end{array}\right) \perp \underbrace{\left(\begin{array}{cc}
A^{-1} \bar{A} & -A^{-1} B \\
C A^{-1} \bar{A}-\bar{C} & D-C A^{-1} B
\end{array}\right)}_{\triangleq_{M_{u b}^{\mu}}^{\mu}(q)}\left(\begin{array}{c}
\lambda^{-} \\
\lambda_{\mathrm{n}, u}
\end{array}\right)+\left(\begin{array}{c}
z_{1} \\
z_{2}
\end{array}\right) \geq 0,
$$

where $A \triangleq A_{n b}-A_{t b}\left[\mu_{b} \xi_{b}\right], \bar{A} \triangleq A_{n b}+A_{t b}\left[\mu_{b} \xi_{b}\right], B \triangleq A_{n b n u}-A_{n b t u}\left[\mu_{u} \xi_{u}\right], C \triangleq A_{\text {nunb }}-$ $A_{\text {nutb }}\left[\mu_{b} \xi_{b}\right], \bar{C} \triangleq A_{\text {nunb }}+A_{\text {nutb }}\left[\mu_{b} \xi_{b}\right], D \triangleq A_{n u}-A_{t u}\left[\mu_{u} \xi_{u}\right], z_{1} \triangleq-A^{-1} w_{1}$, and $z_{2} \triangleq$ $w_{2}+C z_{1}$.

Remark 3 When all the contacts are frictionless, the LCP in (58) has the form

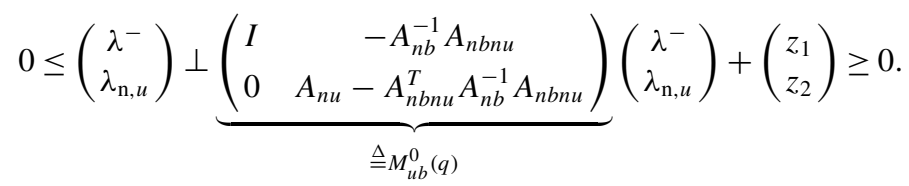

The second line is an LCP involving $\lambda_{n u}$ alone and is the same as the LCP in (31). Thus, its solution $\lambda_{n u}$ exists and is unique, provided that all the constraints are independent. Under this assumption, it follows that $\lambda^{-}$also exists and is unique for any $\left(z_{1}, z_{2}\right)$; hence, the matrix $M_{u b}^{0}(q)$ of the LCP (59) is a P-matrix.

\footnotetext{
${ }^{12}$ Recall $\quad$ that $\quad A_{\text {nunb }}(q) \triangleq \nabla h_{\mathrm{n}, u}(q)^{T} M(q)^{-1} \nabla h_{\mathrm{n}, b}(q), \quad A_{\text {nutb }}(q) \triangleq \nabla h_{\mathrm{n}, u}(q)^{T} M(q)^{-1} H_{\mathrm{t}, b}(q)$, $A_{\text {nbnu }}(q)=A_{\text {nunb }}(q)^{T}, A_{n b t u}(q) \triangleq \nabla h_{\mathrm{n}, b}(q)^{T} M(q)^{-1} H_{\mathrm{t}, u}(q)$.
} 
We have the following decomposition for the matrix in (58): $M_{u b}^{\mu}(q)=M_{u b}^{0}(q)+$ $P_{\mu}(q, \dot{q})$, where $\left\|P_{\mu}(q, \dot{q})\right\|$ tends to zero as the friction coefficient $\mu_{i}$ goes to zero for each contact. Once again, the matrix from the frictional LCP is obtained as a perturbation of the frictionless one (59). Here sufficiently small friction coefficients will guarantee that $M_{u b}^{\mu}$ is a P-matrix. Let us provide some details on the matrix $P_{\mu}(q, \dot{q})$. We know from Sect. 3.2 that $A^{-1} \bar{A}=I+2 K_{\mu}(q, \dot{q})$ with $K_{\mu}(q, \dot{q})=\sum_{i=1}^{\infty}\left(A_{n b}^{-1} A_{t b}\left[\mu_{b} \xi_{b}\right]\right)^{i}$. Using the same decomposition for the other blocks of $M_{u b}^{\mu}(q)$, we obtain:

$$
\begin{aligned}
A^{-1} \bar{A} & =I+2 K_{\mu}(q, \dot{q}), \\
-A^{-1} B= & -A_{n b}^{-1} A_{n b n u}+\left(I+K_{\mu}(q, \dot{q})\right) A_{n b}^{-1} A_{n b t u}\left[\mu_{u} \xi_{u}\right]-K_{\mu}(q, \dot{q}) A_{n b}^{-1} A_{n b n u}, \\
C A^{-1} \bar{A}-\bar{C}= & 0-2 A_{n u t b}\left[\mu_{b} \xi_{b}\right]+C K_{\mu}(q, \dot{q}), \\
D-C A^{-1} B= & A_{n u}-A_{n b n u}^{T} A_{n b}^{-1} A_{n b n u}-A_{t u}\left[\mu_{u} \xi_{u}\right]+A_{n u t b}\left[\mu_{b} \xi_{b}\right] A_{n b}^{-1} A_{n b n u} \\
& +C\left(\left(I+K_{\mu}(q, \dot{q})\right) A_{n b}^{-1} A_{n b t u}\left[\mu_{u} \xi_{u}\right]-K_{\mu}(q, \dot{q}) A_{n b}^{-1} A_{n b n u}\right) .
\end{aligned}
$$

By Theorem 3 in Appendix D, if $\left\|P_{\mu}(q, \dot{q})\right\|_{2}<1 / \beta_{2}\left(M_{u b}^{0}(q)\right)$, then $M_{u b}^{\mu}(q)$ is a P-matrix. We can therefore derive an explicit maximum value of all friction coefficients below that guarantees the existence and uniqueness of a solution to problem (56).

Proposition 13 Let $(q, \dot{q})$ and $F(q, \dot{q}, t)$ be given, and $v_{t, i} \neq 0$ for all $i$. Assume that $M(q) \succ 0$ and $A_{n b}(q) \succ 0$. Suppose that all the constraints are independent, that the bilateral friction coefficients satisfy $\max _{1 \leq i \leq m_{b}} \mu_{i}^{b}<\mu_{\max }^{b}(q)$, and that $\left\|P_{\mu}(q, \dot{q})\right\|_{2}<\frac{1}{\beta_{2}\left(M_{u b}^{0}(q)\right)}$. Then $M_{u b}^{\mu}(q)$ is a P-matrix, and there exists a unique solution $\left(\ddot{q}, \lambda_{n b}, \lambda_{n u}\right)$ to the LCP in (58) for any $z_{1}, z_{2}$ and thus to the mixed sliding friction problem (56).

We see once again that there is no obvious quadratic problem that could be associated with the LCP in (58) because $M_{u b}^{\mu}(q)$ is guaranteed to be neither symmetric nor $\succeq 0$.

Example Proposition 13 defines implicitly a friction bound $\mu_{u b}^{\max }(q)$ below which frictional singularities are avoided. We compute it numerically for the example of a falling ladder in frictional bilateral contact with the wall and frictional unilateral contact with the ground as in Fig. 2(a). The ladder is modeled as rod of length $2 \mathrm{~m}$ and of mass $1 \mathrm{~kg}$. Figure 2(b) depicts the evolution of the friction bound as the rod slides from a vertical position to a horizontal one. It is seen, for example, that at the configuration $\theta=\frac{\pi}{4}$, the friction bound is around 0.65. In Fig. 2(c), we show, for the configuration $\theta=\frac{\pi}{4}$, the range of values $\left(\mu_{b}, \mu_{u}\right)$ of the bilateral and unilateral friction coefficients for which the matrix $M_{u b}^{\mu}(q)$ is a P-matrix. The black region of allowable friction coefficients is thus larger than the safety region defined by the proposition (the $[0,0.65]^{2}$ square). The criterion is thus conservative, as expected.

It is important to mention that the numerical computation of the bound $\mu_{u b}^{\max }(q)$ for a mixed system is not as straightforward as its purely unilateral or purely bilateral counterparts. The quantity $\beta_{2}\left(M_{u b}^{0}(q)\right)$ is by definition the result of an optimization problem (see Theorem D.3 in the Appendix); for the sliding ladder example, we solve it using a sequential least squares solver available in the SciPy library [32]. The matrix $P_{\mu}$ is obtained as the difference $M_{u b}^{\mu}-M_{u b}^{0}$. Finally, the bound $\mu_{u b}^{\max }(q)$ is computed by solving numerically for $\mu$, with a secant method, the scalar algebraic equation $\left\|P_{\mu}(q, \dot{q})\right\|_{2}=1 / \beta_{2}\left(M_{u b}^{0}(q)\right)$. 


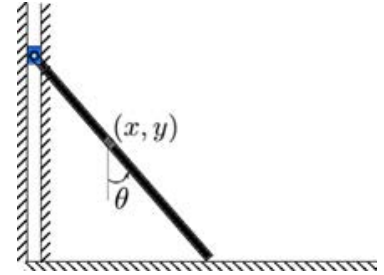

(a) Rod with a Mixed frictional constraints.

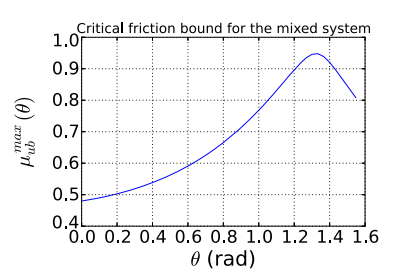

(b) Critical friction bound as a function of the configuration

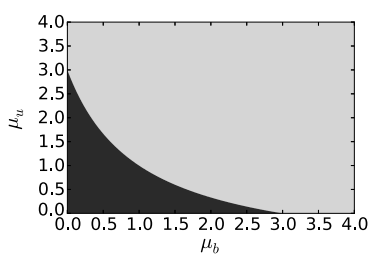

(c) In Black: region of the friction plane where $M_{u b}^{\mu}(q)$ is a P-matrix. Here $\theta=\frac{\pi}{4}$.

Fig. 2 Ladder with two frictional contact points

\subsubsection{Frictionless bilateral contacts}

When the bilateral constraints are frictionless $\left(\mu_{b, i}=0\right.$ for all $\left.0 \leq i \leq m_{b}\right)$, we can easily express $\lambda_{n b}$ as a function of $\lambda_{n u}$, and Eq. (56) becomes

$$
0 \leq \lambda_{n u} \perp\left(D-C A^{-1} B\right) \lambda_{n u}+z_{2} \geq 0
$$

with $z_{2}$ in (58) and $D-C A^{-1} B=A_{n u}-A_{n b n u}^{T} A_{n b}^{-1} A_{n b n u}+\left(A_{n b n u}^{T} A_{n b}^{-1} A_{n b t u}-A_{t u}\right)\left[\mu_{u} \xi_{u}\right]$, which is the sum of the matrix $A_{c}(q)$ in (32) and a frictional perturbation term. We know that $A_{c}(q) \succ 0$ when all constraints are independent (see Sect. 2.3). The matrix $D-C A^{-1} B$ is simpler than $M_{u b}^{\mu}(q)$ in (58) since it is the perturbation of a symmetric positive definite matrix, rather than that of a P-matrix. We can use the results of Sect. 3.3.

Proposition 14 Let $(q, \dot{q})$ and $F(q, \dot{q}, t)$ be given, and $v_{t, i} \neq 0$ for all $i$. Suppose that all constraints are independent, that the bilateral constraints are frictionless $\left(\mu_{b, i}=0\right.$ for all $\left.i\right)$, and that

$$
\max _{1 \leq i \leq m_{u}} \mu_{u, i}<\frac{\sigma_{\min }\left(A_{n u}(q)-A_{n b n u}(q)^{T} A_{n b}(q)^{-1} A_{n b n u}(q)\right)}{\sigma_{\max }\left(A_{t u}(q)-A_{n b n u}(q)^{T} A_{n b}(q)^{-1} A_{n b t u}(q)\right)} .
$$

Then there exists a unique solution $\left(\ddot{q}, \lambda_{n u}, \lambda_{n b}\right)$ to problem (56).

It is worth noting that (64) is the direct extension of (51) taking into account the distortion due to the presence of frictionless bilateral constraints. However, there is no clear relationship between $\mu_{\max }^{u}(q)$ in (51) and the upper bound in (64).

\section{Conclusion}

The main purpose of this article is to present in a progressive way the so-called contact problem (whose unknowns are the contact forces and the accelerations) in the frictionless and sliding Coulomb's friction cases. In both settings, Lagrangian systems with bilateral, unilateral, and mixed bilateral/unilateral constraints are analyzed. Various points of view (linear complementarity problems, inclusions in normal cones, variational inequalities, minimization problems) are considered. The frictional case is treated as a perturbation of the frictionless one, and explicit criteria on the friction coefficients upper bound guaranteeing the well-posedness of a contact problem are given. 


\section{Appendix A: Some convex analysis and complementarity theory tools}

Let $f: \mathbb{R}^{n} \rightarrow \mathbb{R} \cup\{+\infty\}$ be a convex proper and lower semi-continuous function. Its subdifferential, denoted as $\partial f(\cdot)$, is the set of its subgradients. For a set $K$, its indicator function is $\Psi_{K}(x)=0$ if $x \in K$ and $=+\infty$ if $x \notin K$. If $K \subset \mathbb{R}^{n}$ is a nonempty closed convex set, then its normal cone at $x$ is $N_{K}(x)=\left\{z \in \mathbb{R}^{n} \mid z^{T}(y-x) \leq 0\right.$ for all $\left.y \in K\right\}=\partial \Psi_{K}(x)$. If $K=\mathbb{R}^{n}$, then $N_{K}(x)=\{0\}$ for all $x$. The tangent cone $T_{K}(x)=\left\{z \in \mathbb{R}^{n} \mid z^{T} y \leq 0\right.$ for all $\left.y \in N_{K}(x)\right\}$. If $K=\left\{x \in \mathbb{R}^{n} \mid f(x) \geq 0\right\}$ for $m$ continuously differentiable functions $f_{i}: \mathbb{R}^{n} \mapsto \mathbb{R}$ that satisfy the Mangasarian-Fromovitz constraint qualification [19], then $T_{K}(x)=\left\{v \in \mathbb{R}^{n} \mid\right.$ $v^{T} \nabla f_{i}(x) \geq 0$ for all $i \in\{1, \ldots, m\}$ such that $\left.f_{i}(x)=0\right\}$. A linear complementarity problem $\operatorname{LCP}(q, M)$ with unknown $x \in \mathbb{R}^{n}$ is: $x \geq 0, M x+q \geq 0, x^{T}(M x+q)=0$. More compactly, $0 \leq x \perp M x+q \geq 0$. An LCP is said to be solvable if it has at least one solution. A matrix $M$ is a P-matrix if and only if the LCP has a unique solution for any $q$ [16]. A mixed linear complementarity problem (MLCP) is a problem of the form: find vectors $x, y$ such that $M_{11} x+M_{12} y+q_{1}=0$ and $0 \leq x \perp M_{21} x+M_{22} y+q_{2} \geq 0$ for given matrices $M_{i j}$ and vectors $q_{i}$. Let $C$ be a (unnecessarily convex) set of $\mathbb{R}^{n}$; then its dual set is $C^{*}=\left\{x \in \mathbb{R}^{n} \mid x^{T} z \geq 0\right.$ for all $\left.z \in C\right\}$, which is always a closed convex cone. Let $M \in \mathbb{R}^{n \times n}$ be a symmetric positive definite matrix defining the inner product $x^{\mathrm{T}} M x$. With this metric, the orthogonal projection of a vector $x \in \mathbb{R}^{n}$ on a convex set $K \subset \mathbb{R}^{n}$ is denoted as $\operatorname{proj}_{M}[K ; x]=\operatorname{argmin}_{z \in K} \frac{1}{2}(z-x)^{T} M(z-x)$. The following equivalences are useful. Let $x \in \mathbb{R}^{n}, q \in \mathbb{R}^{n}, M \in \mathbb{R}^{n \times n}$, and $K$ be a closed convex cone. Then

$$
\begin{aligned}
M x+q \in-N_{K}(x) & \Leftrightarrow K \ni x \perp M x+q \in K^{*} \\
& \Leftrightarrow \quad\left(\text { if } M=M^{T} \succeq 0\right) \quad x=\operatorname{argmin}_{z \in K} \frac{1}{2} z^{T} M z+q^{T} z \\
& \Leftrightarrow \quad\left(\text { if } M=M^{T} \succ 0\right) \quad x=\operatorname{proj}_{M}\left[K ;-M^{-1} q\right]
\end{aligned}
$$

\section{Appendix B: Theorem 3.1.7 in [16] (excerpts)}

Theorem 1 Let $M \in \mathbb{R}^{n \times n}$ be positive semidefinite, and let $q \in \mathbb{R}^{n}$ be arbitrary. The following statements hold:

(a) If $z^{1}$ and $z^{2}$ are two solutions of the $\operatorname{LCP}(M, q)$, then $\left(z^{1}\right)^{T}\left(q+M z^{2}\right)=\left(z^{2}\right)^{T}\left(q+M z^{1}\right)$.

(d) If $M$ is symmetric (and positive semidefinite), then $M z^{1}=M z^{2}$ for any two solutions $z^{1}$ and $z^{2}$.

\section{Appendix C: Theorem 3.8.6 in [16]}

Theorem 2 Let $M \in \mathbb{R}^{n \times n}$ be copositive, and let $q \in \mathbb{R}^{n}$ be given. If the implication $[0 \leq$ $v \perp M v \geq 0] \Rightarrow\left[v^{T} q \geq 0\right]$ is valid, then the $\operatorname{LCP}(M, q)$ is solvable.

Let $\mathcal{Q}_{M}$ denote the solution set of the homogeneous LCP. This theorem can be restated equivalently as follows: If $M$ is copositive and $q \in \mathcal{Q}_{M}^{*}$, then $\operatorname{LCP}(M, q)$ is solvable. 


\section{Appendix D: Theorems 2.8 and 2.11 in [14]}

Chen and Xiang [14] stated very useful criteria that guarantee that a positive definite or a $\mathrm{P}$-matrix remains positive definite or $\mathrm{P}$ when it is subject to a small enough perturbation. We give here just an excerpt of the results in [14] and a corollary of it.

Theorem 3 If $M$ is a $P$-matrix, then all matrices $A$ such that $\beta_{2}(M)\|M-A\|_{2}<1$ are $P$-matrices, where $\beta_{2}(M):=\max _{d \in[0,1]^{n}}\left\|(I-D+D M)^{-1} D\right\|_{2}$, and $D=\operatorname{diag}(d)$. When $M$ is symmetric positive definite, $\beta_{2}(M)=\left\|M^{-1}\right\|_{2}$.

Theorem 4 Let $M \in \mathbb{R}^{n \times n}$ be a positive definite matrix. Then every matrix $A \in\{A \mid$ $\left.\left\|\left(\frac{M+M^{T}}{2}\right)^{-1}\right\|_{2}\|M-A\|_{2}<1\right\}$ is positive definite.

The next corollary is proved in [10, Corollary 2].

Corollary 3 Let $A=B+C$, where $A, B$ and $C$ are $n \times n$ real matrices, and $B \succ 0$, not necessarily symmetric. If $\|C\|_{2}<\frac{1}{\left\|\left(\frac{B+B^{T}}{2}\right)^{-1}\right\|_{2}}$, then $A \succ 0$.

\section{Appendix E: KKT system: solvability and solution uniqueness}

The KKT problem in (4) is ubiquitous in the study of mechanical systems with bilateral holonomic constraints. In this section, we analyze it from various points of view and we prove that it possesses some subtleties depending on which assumptions are made on the data. Let us consider the next three problems, where $M=M^{T} \in \mathbb{R}^{n \times n}$ and $M \succeq 0, F \in$ $\mathbb{R}^{n \times m}, N \in \mathbb{R}^{n \times m}, x \in \mathbb{R}^{n}, y \in \mathbb{R}^{m}, z \in \mathbb{R}^{n}, a \in \mathbb{R}^{n}, b \in \mathbb{R}^{m}$ :

$$
\begin{aligned}
& \underbrace{\left(\begin{array}{cc}
M & I \\
F^{T} & 0
\end{array}\right)}_{\triangleq \tilde{A} \in \mathbb{R}^{(n+m) \times 2 n}}\left(\begin{array}{l}
x \\
z
\end{array}\right)=\left(\begin{array}{l}
a \\
b
\end{array}\right), \\
& \underbrace{\left(\begin{array}{cc}
M & -F \\
F^{T} & 0
\end{array}\right)}_{\triangleq \bar{A} \in \mathbb{R}^{(n+m) \times(n+m)}}\left(\begin{array}{l}
x \\
y
\end{array}\right)=\left(\begin{array}{l}
a \\
b
\end{array}\right),
\end{aligned}
$$

and

$$
\underbrace{\left(\begin{array}{cc}
M & N \\
F^{T} & 0
\end{array}\right)}_{\triangleq \hat{A} \in \mathbb{R}^{(n+m) \times(n+m)}}\left(\begin{array}{l}
x \\
y
\end{array}\right)=\left(\begin{array}{l}
a \\
b
\end{array}\right) .
$$

The three systems (66), (67), and (68) correspond to various ways to consider the system in (4), where $x$ is for $\ddot{q}, z$ is for $-\nabla h_{\mathrm{n}, b}(q) \lambda_{\mathrm{n}, b}$, and $y$ is for $\lambda_{\mathrm{n}, b}$.

(i) Let us first consider (66) without any assumption on the form of $z$. From [6, Fact 2.10.22] we have $\operatorname{Im}(\tilde{A})=\operatorname{Im}\left(\begin{array}{c}M \\ F^{T}\end{array}\right)+\operatorname{Im}\left(\begin{array}{l}I \\ 0\end{array}\right)$. Thus, a necessary and sufficient condition for (66) to possess a solution $(x, z)$ for any $a$ and $b$, equivalently, $\operatorname{rank}(\tilde{A})=n+m$ (which also follows from [6, Proposition 6.1.7(iii)]), is that $F^{T}$ has full rank $m$, that is, $F$ is of full column rank (this implies that $n \geq m$ ). The uniqueness of $(x, z)$ for any $a$ and $b$ holds if and only if $n=m$ and $\operatorname{rank}(\tilde{\tilde{A}})=2 n$ (this may be 
proved from [6, Theorem 2.6.3(ii)]), in which case, $F$ is square and has full rank $n$. In this case, the solution is equal to $\tilde{A}^{\dagger}\left(\begin{array}{l}a \\ b\end{array}\right)$, where $\tilde{A}^{\dagger}$ is the Moore-Penrose generalized inverse of $\tilde{A}$ [6, Proposition 6.1.7(viii)]. We see that $M$ plays no role in this system.

(ii) Let us still consider (66) assuming that $z=-F y$ for some $y$, that is, $z \in \operatorname{Im}(F)$. We have $(x, z) \in \operatorname{Ker}(\tilde{A}) \Leftrightarrow M x=-z$ and $x \in \operatorname{Ker}\left(F^{T}\right)$. Using $z \in \operatorname{Im}(F)$ and $x \in \operatorname{Ker}\left(F^{T}\right)$ and [6, Theorem 2.4.3], it follows that $x \perp z$. We also have $z \in \operatorname{Im}(M)$. Using that $\operatorname{Im}^{\perp}(M)=\operatorname{Ker}(M)$, we deduce that $x \in \operatorname{Ker}(M)$ because $M$ is symmetric positive semidefinite. ${ }^{13}$ Thus, $x \in \operatorname{Ker}(M) \cap \operatorname{Ker}\left(F^{T}\right)$, and consequently $z=-M x=0$. Thus, we have shown that $[(x, z) \in \operatorname{Ker}(\tilde{A})$ and $z \in \operatorname{Im}(F)] \Rightarrow[z=0$ and $x \in \operatorname{Ker}(M) \cap$ $\left.\operatorname{Ker}\left(F^{T}\right)\right]$, and the reverse implication also holds. Let $S=\left\{(x, z) \in \mathbb{R}^{2 n} \mid z \in \operatorname{Im}(F)\right\}$. Then $\operatorname{Ker}(\tilde{A}) \cap S=\left\{(x, z) \in \mathbb{R}^{2 n} \mid z=0\right.$ and $\left.x \in \operatorname{Ker}(M) \cap \operatorname{Ker}\left(F^{T}\right)\right\}$. We infer that $\operatorname{Ker}(M) \cap \operatorname{Ker}\left(F^{T}\right)=\{0\} \Rightarrow \operatorname{Ker}(\tilde{A}) \cap S=\{0\}$. From (i) existence of solutions for system (66) holds for any $a$ and $b$ if and only if $\operatorname{rank}(\tilde{A})=n+m \Leftrightarrow \operatorname{rank}(F)=m$, hence $\operatorname{dim}(\operatorname{Ker}(\tilde{A}))=2 n-n-m=n-m$. From the fact that $0 \leq \operatorname{dim}(\operatorname{Ker}(\tilde{A}) \cap S) \leq$ $\min (n-m, n+m)=n-m$ [6, Fact 2.9.14], one infers that $\operatorname{Ker}(M) \cap \operatorname{Ker}\left(F^{T}\right)=\{0\} \Rightarrow$ $\operatorname{dim}(\operatorname{Ker}(\tilde{A}) \cap S)=0$, thus $n=m$. In this case it follows from (i) that the system has a unique solution for any $a$ and $b$. Conversely the existence of solutions for arbitrary $a$ and $b$ and $n=m$ imply uniqueness, as well as $\operatorname{Ker}(M) \cap \operatorname{Ker}\left(F^{T}\right)=\{0\}$ since $F$ is square full rank $n$.

Let us now pass to the system (67). Remark that if $\bar{A}$ in (67) is invertible then $F$ necessarily has full rank $m$. This follows from the fact that $(x, y) \in \operatorname{Ker}(\bar{A}) \operatorname{implies} x \in \operatorname{Ker}(M) \cap$ $\operatorname{Ker}\left(F^{T}\right)$ and $y \in \operatorname{Ker}(F)$, using similar arguments as in (ii). In particular, if there are more constraints than degrees of freedom (i.e., $m>n$ ), then $\bar{A}$ is not invertible, and likewise if $\operatorname{rank}(F)=r<m$.

(iii) Consider now system (67). Let $\operatorname{rank}(F)=m$ (so $m \leq n$ ), and $M$ be positive semidefinite. Then the existence and uniqueness of both $x$ and $y$ for arbitrary $a$ and $b$ (equivalently, nonsingularity of $\bar{A}$ ) holds if and only if $\operatorname{Ker}(M) \cap \operatorname{Ker}\left(F^{T}\right)=\{0\}$ (proof by direct application of [8, p. 523] or by using the above expression of $\operatorname{Ker}(\bar{A}))$.

The rank condition on $F$ appears to be in fact necessary and sufficient as alluded to few lines above:

(iii') System (67) has a unique solution $(x, y)$ for arbitrary $a$ and $b$ if and only if $\operatorname{rank}(F)=$ $m$ and $\operatorname{Ker}(M) \cap \operatorname{Ker}\left(F^{T}\right)=\{0\}$. This solution is equal to $\bar{A}^{-1}\left(\begin{array}{l}a \\ b\end{array}\right)$.

The proof of (iii') follows from [6, Theorem 2.6.3, Proposition 6.1.7], noting that $\bar{A}$ is square. It is sometimes wrongly stated that $\bar{A}$ is nonsingular if and only if $M$ and $F$ are both full-rank matrices [34], which is only a sufficient condition. In fact, from [6, Fact 6.4.20] we have:

$$
\begin{aligned}
\operatorname{rank}(\bar{A})= & \operatorname{rank}(M)+2 \operatorname{rank}(F)-\operatorname{dim}[\operatorname{Im}(M) \cap \operatorname{Im}(F)] \\
& -\operatorname{dim}\left[\operatorname{Im}\left(\begin{array}{c}
M \\
F^{T}
\end{array}\right) \cap \operatorname{Im}\left(\begin{array}{c}
F \\
0
\end{array}\right)\right] .
\end{aligned}
$$

Formula (69) shows that we may dispense with positive definiteness conditions on $M$ and that the nonsingularity of $\bar{A}$ results from an interplay between the matrices ranges. Consider, for instance, $M=\left(\begin{array}{cc}1 & 0 \\ 0 & -1\end{array}\right)$ and $F=\left(\begin{array}{ll}1 & 0\end{array}\right)^{T}$, which yields $\operatorname{rank}(\bar{A})=3$. In contact mechanics, we wish to allow for situations where the constraints are redundant, so that $(\operatorname{rank}(F)=r<$

\footnotetext{
${ }^{13}$ The conclusion does not hold without the positive definiteness condition.
} 
$\min (m, n))$, but which are nevertheless compatible (i.e., $\left.b \in \operatorname{Im}\left(F^{T}\right)\right)$ since otherwise the problem has no solution. Thus, the most relevant problem is that of determining $x$ and $y$ such that (67) holds for arbitrary $a$ with the additional assumption that $b \in \operatorname{Im}\left(F^{T}\right)$. This problem is thus different from problems tackled in (ii) and (iii') and corresponds to the problem tackled in [30, 31, 59].

(iv) The necessary and sufficient condition for the existence of $x$ and $y$ with uniqueness of $x$ and $F y$ such that (67) holds for arbitrary $a$ is that $b \in \operatorname{Im}\left(F^{T}\right)$ and $\operatorname{Ker}(M) \cap \operatorname{Ker}\left(F^{T}\right)=$ $\{0\}$.

\section{Proof}

$\Leftarrow$

Existence of $x$ and $y$ : By contraposition, if not ( $\forall a, \exists x$ and $y$ such that (67) holds), then there exists $a \in \mathbb{R}^{n}$ such that for all $x, y, F^{T} x \neq b$ (so $b \notin \operatorname{Im}\left(F^{T}\right)$ ) or $M x-F y \neq a$ (so $\operatorname{Im}([M F]) \neq \mathbb{R}^{n}$, that is, $\operatorname{Ker}(M) \cap \operatorname{Ker}\left(F^{T}\right) \neq\{0\}$.

Uniqueness of $x$ and $F y$ : Suppose that $b \in \operatorname{Im}\left(F^{T}\right)$ and $\operatorname{Ker}(M) \cap \operatorname{Ker}\left(F^{T}\right)=\{0\}$.

If $\left(x_{1}, y_{1}\right)$ and $\left(x_{2}, y_{2}\right)$ are two solutions of (67), then $F^{T}\left(x_{1}-x_{2}\right)=0$ and $M\left(x_{1}-x_{2}\right)=$ $F\left(y_{1}-y_{2}\right)$. Hence, $\left(x_{1}-x_{2}\right) \in \operatorname{Ker}\left(F^{T}\right)$ and $M\left(x_{1}-x_{2}\right) \in \operatorname{Im}(F)=\operatorname{Ker}\left(F^{T}\right)^{\perp}$. Hence, $\left(x_{1}-x_{2}\right)^{T} M\left(x_{1}-x_{2}\right)=0$, and since $M$ is symmetric positive semidefinite, this means $\left(x_{1}-x_{2}\right) \in \operatorname{Ker}(M)$. By hypothesis, $\operatorname{Ker}(M) \cap \operatorname{Ker}\left(F^{T}\right)=\{0\}$, so we conclude that $x_{1}-x_{2}=$ 0 , that is, $x$ is unique. It follows that $F y_{1}=F y_{2}$, so that $F y$ is unique as well.

$\Rightarrow$

Suppose that for arbitrary $a$, there exist $x$ and $y$ such that (67) holds with uniqueness of $x$ and $F y$. Then, in particular, $b \in \operatorname{Im}\left(F^{T}\right)$. Let $x \in \operatorname{Ker}(M) \cap \operatorname{Ker}\left(F^{T}\right)$, and let $\left(x^{\star}, y\right)$ be the unique solution of (67). Then $\left(x^{\star}+x, y\right)$ is also a solution of (67). Hence, $x=0$, so that $\operatorname{Ker}(M) \cap \operatorname{Ker}\left(F^{T}\right)=0$.

In [31, p. 319], the condition $b \in \operatorname{Im}\left(F^{T}\right)$ is stated as follows: acceleration constraints are compatible. In conclusion, four types of systems are considered: system (67) with unknowns $x$ and $y$ in (iii), system (66) with unknowns $x$ and $z$ in (i), system (66) with unknowns $x$ and $z$ and the constraints that $z \in \operatorname{Im}(F)$ in (ii), system (67) with unknowns $x$ and $y$ with uniqueness of $x$ and $F y$ and arbitrary $a$ in (iv). To complete the picture, let us note that $\operatorname{Ker}(M) \cap \operatorname{Ker}\left(F^{T}\right)=\{0\} \Leftrightarrow(\operatorname{Im}(M)+\operatorname{Im}(F))=\mathbb{R}^{n} \Leftrightarrow \operatorname{Im}[(M F)]=\mathbb{R}^{n}$ using [6, Fact 2.9.10], and we recover directly an alternative way to formulate the condition involving the kernels, sometimes used in the literature [59] [30, Eq. (9)].

Problems like in (67) and (66) occur in frictionless systems. The next step is to consider systems of the form (68) for some matrix $N$. Such problems arise in the presence of Coulomb's friction; see (36). Using [6, Fact 6.4.20], we get an extension of (69):

$$
\begin{aligned}
\operatorname{rank}(\hat{A})= & \operatorname{rank}(M)+\operatorname{rank}\left(F^{T}\right)+\operatorname{rank}(N)-\operatorname{dim}[\operatorname{Im}(M) \cap \operatorname{Im}(F)] \\
& -\operatorname{dim}\left[\operatorname{Im}\left(\begin{array}{c}
M \\
F^{T}
\end{array}\right) \cap \operatorname{Im}\left(\begin{array}{c}
N \\
0
\end{array}\right)\right] .
\end{aligned}
$$

System (68) has a unique solution $(x, y)$ for any $a$ and $b$ if and only if $\operatorname{rank}(\hat{A})=n+m$. We have:

$$
\begin{aligned}
& \operatorname{Im}\left(\begin{array}{c}
M \\
F^{T}
\end{array}\right) \cap \operatorname{Im}\left(\begin{array}{c}
N \\
0
\end{array}\right) \\
& \quad=\left\{z \in \mathbb{R}^{n+m} \mid \exists y_{1} \in \operatorname{Ker}\left(F^{T}\right), \exists y_{2} \in \mathbb{R}^{m} \text { such that } z=\left(\begin{array}{c}
M y_{1} \\
0
\end{array}\right)=\left(\begin{array}{c}
N y_{2} \\
0
\end{array}\right)\right\}
\end{aligned}
$$


and $\operatorname{Im}(M) \cap \operatorname{Im}(F)=\left\{z \in \mathbb{R}^{n} \mid \exists y_{1} \in \mathbb{R}^{n}, \exists y_{2} \in \mathbb{R}^{m}\right.$ such that $\left.z=M y_{1}=F y_{2}\right\}$. It is clear from (70) that the well-posedness of a system depends on the interplay between $M, F$, and $N$. Even if all three matrices have full rank, then we may have $\operatorname{rank}(\hat{A})<$ $n+m$. Suppose that $\operatorname{rank}(M)=n$, and $\operatorname{rank}\left(F^{T} M^{-1} N\right)=m$ (implying that $\left.m \leq n\right)$. Then $\operatorname{Im}(M) \cap \operatorname{Im}(F)=\operatorname{Im}(F)$ and $\operatorname{Im}\left(\begin{array}{c}M \\ F^{T}\end{array}\right) \cap \operatorname{Im}\left(\begin{array}{c}N \\ 0\end{array}\right)=\{0\}$ (since $F^{T} y_{1}=0=F^{T} M^{-1} N y_{2}$ ). Therefore, from (70) we have $\operatorname{rank}(\hat{A})=n+m$.

Finally, we may rewrite (68) as (66) posing $z=N y$. Then (i) applies, but (ii) usually is not except if $\operatorname{Im}(N) \subseteq \operatorname{Im}(F)$. Then given $z$, there exists a unique $y$ if and only if $N$ has full column rank $m(\Rightarrow m \leq n)$. Let $N=-F+P$ for some matrix $P$.

(v) Assume that $F$ has full column rank $m$ (equivalently, $F^{T} F \in \mathbb{R}^{m \times m}$ is positive definite). Let us investigate conditions that guarantee that $N$ has full rank $m$ (equivalently, $N^{T} N \in$ $\mathbb{R}^{m \times m}$ is positive definite). We have $N^{T} N=F^{T} F-F^{T} P-P^{T} F+P^{T} P$. A direct application of Corollary 3, with matrices $A, B, C$ chosen as $A=N^{T} N, B=F^{T} F$ and $C=-F^{T} P-P^{T} F+P^{T} P$, shows that a sufficient condition for $N^{T} N$ to be positive definite is that $\left\|-F^{T} P-P^{T} F+P^{T} P\right\|_{2}<\frac{1}{\left\|\left(F^{T} F\right)^{-1}\right\|_{2}}$ or, equivalently, $\sigma_{\max }\left(-F^{T} P-\right.$ $\left.P^{T} F+P^{T} P\right)<\sigma_{\min }\left(F^{T} F\right)$.

Let $M=0$. Then, using (70), it follows that $\operatorname{rank}(\hat{A})=2 m$; hence, $\operatorname{rank}(\hat{A})=n+m$ if and only if $n=m$. This shows that, depending on the interplay between the ranges of the matrices in (70), system (68) may be solvable with uniqueness for any $a$ and $b$, for low-rank matrices $M$.

(vi) Let us assume that $\operatorname{rank}(M)=n$ and study conditions such that the rank of $F^{T} M^{-1} N=$ $-F^{T} M^{-1} F+F^{T} M^{-1} P$ is $m$. Then, as shown after (71), $\hat{A}$ has rank $n+m$, and system (68) has a unique solution for any $a$ and $b$. Using Corollary 3 with matrices $A, B, C$ chosen as $A=-F^{T} M^{-1} N, B=F^{T} M^{-1} F$ and $C=-F^{T} M^{-1} P$, we conclude that $\operatorname{rank}\left(F^{T} M^{-1} N\right)=m$ if $\operatorname{rank}(F)=m$ and $\sigma_{\max }\left(-F^{T} M^{-1} P\right)<\sigma_{\min }\left(F^{T} M^{-1} F\right)$.

It is worth noting that the study of problem (68) may also be quite useful in the context of numerical analysis of differential algebraic equations (DAEs). Half-explicit methods involve such problems (for instance, $N$ may be the Jacobian of the constraints estimated at step $i+1$, whereas $F$ is the Jacobian estimated at step $i$ ) [56, §7.1], [24, §VII.6]; see also [13, 39, 45, 46] for various forms of numerical KKT systems.

\section{References}

1. Acary, V., Brogliato, B.: Numerical Methods for Nonsmooth Dynamical Systems. Lecture Notes in Applied and Computational Mechanics, vol. 35. Springer, Berlin (2008). doi:10.1007/978-3-540-75392-6

2. Acary, V., Brogliato, B., Goeleven, D.: Higher order Moreau's sweeping process: mathematical formulation and numerical simulation. Math. Program. A 113(1), 133-217 (2008). doi:10.1007/s10107-0060041-0

3. Addi, K., Brogliato, B., Goeleven, D.: A qualitative mathematical analysis of a class of linear variational inequalities via semi-complementarity problems. Applications in electronics. Math. Program. A 126(1), 31-67 (2011). doi:10.1007/s10107-009-0268-7

4. Anh, L.X.: Dynamics of Mechanical Systems with Coulomb Friction. Foundations of Mechanical Engineering. Springer, Berlin (2003)

5. Bayo, E., Ledesma, R.: Augmented Lagrangian and mass-orthogonal projection methods for constrained multibody dynamics. Nonlinear Dyn. 9(1-2), 113-130 (1996). doi:10.1007/BF01833296

6. Bernstein, D.: Matrix, Mathematics. Theory, Facts, and Formulas with Application to Linear Systems Theory. Princeton University Press, Princeton (2005)

7. Blajer, W.: Augmented Lagrangian formulation: geometrical interpretation and application to systems with singularities and redundancy. Multibody Syst. Dyn. 8(2), 141-159 (2002) 
8. Boyd, S., Vandenberghe, L.: Convex Optimization. Cambridge University Press, Cambridge (2004)

9. Brogliato, B.: Inertial couplings between unilateral and bilateral holonomic constraints in frictionless Lagrangian systems. Multibody Syst. Dyn. 29, 289-325 (2013). doi:10.1007/s11044-012-9317-8

10. Brogliato, B.: Kinetic quasi-velocities in unilaterally constrained Lagrangian mechanics with impacts and friction. Multibody Syst. Dyn. 32, 175-216 (2014). doi:10.1007/s11044-013-9392-5

11. Brogliato, B., Goeleven, D.: Singular mass matrix and redundant constraints in unilaterally constrained Lagrangian and Hamiltonian systems. Multibody Syst. Dyn. 35, 39-61 (2015). doi:10.1007/s11044-0149437-4

12. Brogliato, B., Thibault, L.: Existence and uniqueness of solutions for non-autonomous complementarity dynamical systems. J. Convex Anal. 17(3-4), 961-990 (2010)

13. Brüls, O., Arnold, M.: Convergence of the generalized $\alpha$ scheme for constrained mechanical systems. Multibody Syst. Dyn. 18(2), 185-202 (2007)

14. Chen, X., Xiang, S.: Perturbation bounds of P-matrix linear complementarity problems. SIAM J. Optim. 18(4), 1250-1265 (2007)

15. Cottle, R.: On a problem in linear inequalities. J. Lond. Math. Soc. 1(1), 378-384 (1968)

16. Cottle, R., Pang, J., Stone, R.: The Linear Complementarity Problem. Computer Science and Scientific Computing. Academic Press, San Diego (1992)

17. ten Dam, A., Dwarshuis, E., Willems, J.: The contact problem for linear continuous-time dynamical systems: a geometric approach. IEEE Trans. Autom. Control 42(4), 458-472 (1997)

18. Dopico, D., González, F., Cuadrado, J., Kövecses, J.: Determination of holonomic and nonholonomic constraint reactions in an index-3 augmented Lagrangian formulation with velocity and acceleration projections. J. Comput. Nonlinear Dyn. 9(4), 041006 (2014)

19. Facchinei, F., Pang, J.: Finite-Dimensional Variational Inequalities and Complementarity Problems. Operations Research, vol. 1. Springer, New York (2003)

20. Fraczek, J., Wojtyra, M.: On the unique solvability of a direct dynamics problem for mechanisms with redundant constraints and Coulomb friction in joints. Mech. Mach. Theory 46(3), 312-334 (2011). doi: 10.1016/j.mechmachtheory.2010.11.003

21. Génot, F., Brogliato, B.: New results on Painlevé paradoxes. Eur. J. Mech. A, Solids 18(4), 653-677 (1999). doi:10.1016/S0997-7538(99)00144-8

22. Glocker, C.: The principles of d'Alembert, Jourdain, and Gauss in nonsmooth dynamics, part I: scleronomic multibody systems. Z. Angew. Math. Mech. 78, 21-37 (1998)

23. Glocker, C.: Set-Valued Force Laws: Dynamics of Non-Smooth Systems. Springer, Berlin (2001)

24. Hairer, E., Wanner, G.: Solving Ordinary Differential Equations II. Stiff and Differential-Algebraic Problems. Series in Computational Mathematics, vol. 14. Springer, Berlin (1996), second revised edn.

25. Hjiaj, M., de Saxcé, G., Mroz, Z.: A variational inequality-based formulation of the frictional contact law with a non-associated sliding rule. Eur. J. Mech. A, Solids 21, 49-59 (2002)

26. Hurtado, J., Sinclair, A.: Lagrangian mechanics of overparameterized systems. Nonlinear Dyn. 66, 201$212(2011)$

27. Ivanov, A.: Singularities in the dynamics of systems with non-ideal constraints. J. Appl. Math. Mech. 67(2), 185-192 (2003)

28. Izmailov, A., Kurennoy, A., Solodov, M.: Local convergence of the method of multipliers for variational and optimization problems under the noncriticality assumption. Comput. Optim. Appl. 1(30), 111-140 (2014). doi:10.1007/s10589-014-9658-8

29. Jain, A.: Operational space for closed-chain robotic systems. ASME J. Comput. Nonlinear Dyn. 9(2), 021015 (2014). doi:10.1115/1.4025893

30. de Jalon, J.G., Callejo, A., Hidalgo, A.: Efficient solution of Maggi's equations. ASME J. Comput. Nonlinear Dyn. 7, 021003 (2012)

31. de Jalon, J.G., Gutierrez-Lopez, M.: Multibody dynamics with redundant constraints and singular mass matrix: existence, uniqueness, and determination of solutions for accelerations and constraints forces. Multibody Syst. Dyn. 30(3), 311-341 (2013). doi:10.1007/s11044-013-9358-7

32. Jones, E., Oliphant, T., Peterson, P., et al.: SciPy: open source scientific tools for Python (2001-). URL http://www.scipy.org/. [Online; accessed 2016-05-04]

33. Lancaster, P., Tismenetsky, M.: The Theory of Matrices, 2nd edn. Academic Press, San Diego (1985)

34. Laulusa, A., Bauchau, O.: Review of classical approaches for constraint enforcement in multibody systems. ASME J. Comput. Nonlinear Dyn. 3(1), 011004 (2008)

35. Leine, R., Brogliato, B., Nijmeijer, H.: Periodic motion and bifurcations induced by the Painlevé paradox. Eur. J. Mech. A, Solids 21(5), 869-896 (2002). doi:10.1016/S0997-7538(02)01231-7. http://www. sciencedirect.com/science/article/pii/S0997753802012317

36. Leine, R., van de Wouw, N.: Stability and Convergence of Mechanical Systems with Unilateral Constraints. Lecture Notes in Applied and Computational Mechanics, vol. 36. Springer, Berlin (2008) 
37. Lötstedt, P.: Coulomb friction in two-dimensional rigid body systems. Z. Angew. Math. Mech. 61, 605615 (1981)

38. Lötstedt, P.: Mechanical systems of rigid bodies subject to unilateral constraints. SIAM J. Appl. Math. 42(2), 281-296 (1982)

39. Lunk, C., Simeon, B.: Solving constrained mechanical systems by the family of Newmark and $\alpha-$ methods. J. Appl. Math. Mech., Z. Angew. Math. Mech. 86(10), 772-784 (2006)

40. Anitescu, M., Potra, F.A.: A time-stepping method for stiff multibody dynamics with contact and friction. Int. J. Numer. Methods Eng. 55(7), 753-784 (2002). doi:10.1002/nme.512

41. Matrosov, V., Finogenko, I.: Right-hand solutions of the differential equations of dynamics for mechanical systems with sliding friction. J. Appl. Math. Mech. 59(6), 837-844 (1995)

42. Matrosov, V.M., Finogenko, I.: The theory of differential equations which arise in the dynamics of a system of rigid bodies with Coulomb friction. Monogr. Acad. Nonlinear Sci., Adv. Nonlinear Sci. 2, $16-106$ (2008)

43. Moreau, J.: Les liaisons unilatérales et le principe de Gauss. C. R. Acad. Sci. 256(4), 871-874 (1963)

44. Moreau, J.: Quadratic programming in mechanics: dynamics of one-sided constraints. SIAM J. Control 4(1), 153-158 (1966)

45. Murua, A.: Partitioned half-explicit Runge-Kutta methods for differential-algebraic systems of index 2. Computing 59(1), 43-61 (1997)

46. Negrut, D., Jay, L., Khude, N.: A discussion of low-order numerical integration formulas for rigid and flexible multibody dynamics. ASME J. Comput. Nonlinear Dyn. 4, 021008 (2009)

47. Painlevé, P.: Leçons sur le Frottement. Hermann, Paris (1895)

48. Pang, J., Trinkle, J.: Complementarity formulation and existence of solutions of dynamic rigid-body contact problems with Coulomb friction. Math. Program. 73(2), 199-226 (1996)

49. Pang, J., Trinkle, J., Lo, G.: A complementarity approach to a quasistatic multi-rigid-body contact problem. J. Comput. Optim. Appl. 5(2), 139-154 (1996)

50. Pfeiffer, F.: On non-smooth multibody dynamics. Proc. Inst. Mech. Eng., Part K, J. Multi-Body Dyn. 226(2), 147-177 (2012)

51. Rockafellar, R.T.: Convex Analysis, vol. 28. Princeton University Press, Princeton (1970). doi:10.1142/ 9789812777096

52. Ruzzeh, B., Kövecses, J.: A penalty formulation for dynamics analysis of redundant mechanical systems. J. Comput. Nonlinear Dyn. 6(2), 021008 (2011)

53. de Saxcé, G., Feng, Z.: New inequality and functional for contact friction: the implicit standard material approach. Mech. Struct. Mach. 19, 301-325 (1991)

54. van der Schaft, A., Schumacher, J.: Complementarity modeling of hybrid systems. IEEE Trans. Autom. Control 43(4), 483-490 (1998)

55. Shabana, A.: Euler parameters kinetic singularity. Proc. Inst. Mech. Eng., Part K, J. Multi-Body Dyn. (2014). doi:10.1177/1464419314539301

56. Simeon, B.: Computational Flexible Multibody Dynamics. A Differential-Algebraic Approach. Springer, Berlin (2013). Differential-Algebraic Equations Forum

57. Terze, Z., Müller, A., Zlatar, D.: Lie-group integration method for constrained multibody systems in state space. Multibody Syst. Dyn. 34(3), 275-305 (2015). doi:10.1007/s11044-014-9439-2

58. Udwadia, F., Kalaba, R.: Analytical Dynamics: A New Approach. Cambridge University Press, Cambridge (1996)

59. Udwadia, F., Phohomsiri, P.: Explicit equations of motion for constrained mechanical systems with singular mass matrices and applications to multi-body dynamics. Proc. R. Soc. Lond. A 462, 2097-2117 (2006)

60. Udwadia, F., Schutte, A.: Equations of motion for general constrained systems in Lagrangian mechanics. Acta Mech. 213(1-2), 111-129 (2010)

61. Wojtyra, M.: Joint reactions in rigid body mechanisms with dependent constraints. Mech. Mach. Theory 44(12), 2265-2278 (2009). doi:10.1016/j.mechmachtheory.2009.07.008

62. Wojtyra, M., Fraczek, J.: Comparison of selected methods of handling redundant constraints in multibody systems simulations. ASME J. Comput. Nonlinear Dyn. 8, 0210007 (2013)

63. Wojtyra, M., Fraczek, J.: Solvability of reactions in rigid multibody systems with redundant nonholonomic constraints. Multibody Syst. Dyn. 30(2), 153-171 (2013). doi:10.1007/s11044-013-9352-0 